CONTENTS

Acknowledgments . . . . . . . . . . . . . . . vii Abstract .................. . . . . ix

I. Introduction .... . . . . . . . . . 1

II. Strong Turbulence Theory . . . . . . . . . . . 5

III. The Random Phase Approximation . . . . . . . . 9

IV. Conversion to Integral Equation . . . . . . . 17

v. Uniform Beams . . . . . . . . . . . . 20

VI . Integral Equation for Bunched Beams . . . . . . 22

VII. Bunched Proton Beams Without Turbulence . . . . 26

VIII. Electron Beams . . . . . . . . . . 36

1X. Comparison With Experiments . . . . . . . . . 42

X. Summary and Conclusions . . . . . . . . . 51

Appendix A . . . . . . . . . . . . 54

Appendix B ................... 57

Appendix C................... 61

Appendix D . . . . . . . . . . . . . 64

References . . . . . . . . . . . 74

Figure Captions . . . . . . . . . . . 76

Figures .................. 78 


\section{DEDICATION}

for Dianne Sancic-Channel1 


\section{ACKNOWLEDGMENTS}

I want to thank A. M. Sessler for his guidance of, and constant involvement in, every aspect of this thesis. I want to thank L. J. Laslett for conversations about this work and for help in programming; and J. M. Paterson for providing me with the data from SPEAR II and for discussing it with me. 


\title{
STRONG TURBULENCE AND THE ANOMALOUS \\ LENGTH OF STORED PARTICLE BEAMS
}

Paul J. Channe11

\author{
Lawrence Berkeley Laboratory \\ University of California \\ Berkeley, California 94720
}

November 1975

\begin{abstract}
A theoretical analysis is made of the longitudinal stability of intense beams of charged particles in high energy storage rings. The effect of externally applied radiofrequency fields, i.e., synchrotron oscillations, is included. For electron and positron beams, the damping and quantum excitation due to particle radiation are also included. A criterion for the stability of small longitudinal plasma oscillations is derived and used to obtain the stable size of intense stored beans of electrons and positrons.

Anomalous lengthening of stored electron and positron beams has been experimentally measured. Previous theories have assumed that the effect is due to modification of the potential-well due to self-fields or to the excitation of coherent synchrotron oscillations. One previous calculation assumed that the effect was due to strongly turbulent collective motion, but failed to take into account the effect of the external radiofrequency fields, i.e., bunching. We calculate the bunch lengthening under
\end{abstract}


the assumption that turbulent oscillations are present and include the effect of bunching and associated synchrotron oscillations. We also include the effect of the rudification of the potential-well due to self-fields.

Since strong turbulence is assumed to be partially responsible for bunch lengthening, we first derive, using a random phase approximation, the basic equation of one-dimensional strong turbulence theory. It is then shown, using a criterion due to Chirikov, that particle trajectories are stochastic and that this leads to the mixing of wave phases assumed in the random phase approximation.

The dispersion relation for longitudinal perturbations of turbulent uniforn beams is derived and compared with previous results. The integral equation for perturbations of bunched proton beams without turbulence is derived, and the dispersion relation for the short-wavelength case is given. Landau darming is included in an approximate frshion.

The integral equation for perturbations of electron and positron beams is presented. The dispersion relation for short-wavelength perturtations is derived. By assuming that the level of turbulence will increase to a point where small perturbations are stable, a formula for bunch length is derived which, typically, gives values which are greater than the natural bunch length and which depend on current.

The formula for bunch length is compared with experimental data from SPEAR I and from SPEAR II. The data from SPEAR I can be fit very well if sizeable contributions from both turbulence and self-field distortion of the potential well are included. The data from SPEAR II can be fit very well by only including the effect of turbulence, in 
prot-isle accurd with the absence of ferrite magnets in SPEAR II. The possible relation of a saturation phemomenon, observed on SPEAR II, to norlinizar potentiall well distortion and to mode-mode coupling is discussed. 


\section{INTRONUCTION}

In the presence of radiofrequency fieIds, charged particles in storage rings, with energies different from the average energy, undergo longitudinal synchrotron oscillations about a synchronous orbit. Flectrons and positrons, in addition, radiate significantly, and this tends to damp out the synchrotron oscillations. However, because the radiation is quantum mechanical, it is "noisy", and this noise causes diffusion. The natural length of electron and positron beams is due to the balance of radiation damping with quantum diffusion and is easily calculated. Charged particles also undergo transverse oscillations, betatron oscillations, about a "design" orbit as a result of magnetic focusing forces. For electrons and positrons, radiation damping and quantum excitation determine part of the natural width for the beans. However, because the radial position o: a charged particle depends on energy, the energy spread of the beam contributes to the width. Given the parameters of the particular nachine, the natural bunch width can be easily calculated.

It has been observed at Orsay, Frascati, ${ }^{1}$ and at Stanford, both with SPEAR $I^{2}$ and with SPEAR II, ${ }^{3}$ that bunches are anomalously long and that this effect depends on the total current in the bunch. In addition, experiments have shown that the effect is independent of the number of bumches present. Thus, the effect seems to be local.

With SPEAR II, an anmalous, current-dependent widening has also been measured. ${ }^{j}$ This suggests that an increase in energy spread may contribute to the bunch lengthening phenomenon. A threshcld in current has also been observed.

These observations have stimulated considerable theoretical effort, 
and three types of theories have been developed. In the first type, the equilibrium theories, the bunch lengthening effect is assumed to he due to the modification of the potential well that individual particles see by the self-fields of the bunch. A general formulation of these theories in linear approximation has been developed by Pellegrini and Sessler. More recently, the effect on the bunch length of the nonlinear distortion of the potential well due to self-fields has been considered by Keil. ${ }^{5}$ These theories have dependences on current, heam energy, and $\mathrm{RF}$ voltage that are roughly in agreement with the data. However, because self-fields are present even at very small currents, these theories cannot account for the observed threshold effect in current. In addition, these theories predict no increase in energy widch, and thus cannot account for the observed bunch widening.

The second type of theory, due to Lebedev, ${ }^{6}$ assumes that bunch lengthening is due to the excitation of coherent synchrotron oscillations in the bunch. In response, the energy spread of the bunch incrcases until the coherent oscillations are stabilized. In this theory, the effect has dependences on current and $R F$ voltage which are in reasonable agreement with the data. In addition, the threshold effect and bunch widening are easily explained by this theory. The threshold effect is simply due to the fact that below a certain current the beam is naturally stable against coherent oscillations. The bunch widening is due to the increase in energy spread. However, this theory has too strong a dependence on beam energy to fit the data.

The thind type of theory, related to the second type, was developed by Sessler. ${ }^{7}$ In this theory, if the bunch is unstable against coherent oscillations, a turbulent state develops which increases the diffusion 
coefficient, and thus the energy width, until the oscillations are stabilized. The lengthened steady state is thus assumed to be turbulent. The stability criterion is calculated using the strong turbulence theory of Dupree ${ }^{8}$ and others. ${ }^{9-1]}$ However, the calculation was done for a uniform, i.e., unbunched, beam, and the results were applied in an ad hoc manner to bunched beams. Thus, although the fits to the data were fairly good, the need for a calculation for bunched beams was evident. In fact, we will see that the good fit to the data that this theory gave was, to a certain extent, fortuitous.

In this thesis, we calculate the bunch lengthening effect for bunched beans by using strong turhulence theory. In addition, we include the equilibrium effect, i.e., the potential well distortion due to self-fields. We find that the data from SPEAR 1 can be fit very well only if we include large contrihutions from both turbulence and equilibrium effects. The SPFAR II data, on the other hand, can be fit hy assuming only turbulence. This is consistent with the observation of a threshold in current at SPEAR 1I. In addition, the absence of equilibrium ef fect for SPFAR II is reasonable when the differences hetween SPEAR I and SPEAR II are taken into account, i.e., the lack of ferrite kicker magnets and the different RF system for SPEAR II.

As can be seen, our calculation encompasses all three previous types of theories. An effect which is not explicitly included in our calculation is the nonlinear distortion of the potential well due to self-fields. We will comment on the possible contribution of this when we examine the data from SPEAR II. Also not included is the effect of mode-mode coupling, bu: we will coment on its possible contribution. In Section II, we derive the strong turbulence theory by a Fokker- 
Planck technique. Although our derivation is considerably less general than other derivations, the calculation serves to point out the plysical assunptions involved anci establishes the basic equation which we use. In Section 1II, the raudom phase approximation, wheh was used in deriving the strong turbulene? theory, is examined. In particular, it is argucd that, under reasonable physical circumstances, trajectorics of particles interacting with short wavelength waves are stcchastic, i.e., random, and that this leads to a mixing of wave phases.

In Section IV, we convert our integro-differential equation into an integral equation. In Section $V$, tr is integral equation is used to derive the dispersion relation for uniform beams. Thus, ve reproduce Sessler's previous result. ${ }^{7}$ In Section $W_{i}$, we convert our integral equation into a simpler integral equation in the case of bunched beams. In Section VII, this integral equation is applied to proton beams. A1so, the Landau damping due to nonlinear potential wells is discussed.

In Section VIII, the electron beam case is solved and the dispersion relation is obtained. From this, a formula for the bunch length follows. In Section IX, our formula for bunch length is compared with experimental data from SPFAR I and from SPEAR II. He discuss possible additional effects and suggest experiments to clarify the situation. In Section $X$, we summarize our results and present our conclusions.

In Appendices $A$ and $B$, some relevant mathematical calculations are presented. In Appendix $C$, an alternate derivation for the uniform bean case is presented, and the various results are shown to agrec with each other. In Appendix D, a function necessary for the electron beam case is calculated. 


\section{STRONG TURBULENCE THEORY}

In this section we derive our basic equation from strong turbulence theory. Weak turbulence is characterized by the fact that the time for a particle to diffuse through a typical wavelength, 'TR in Duprce's notation, ${ }^{8}$ is long compared to the autocorrelation time, $\tau_{A C}$, of the turbulent electric field; thus $\tau_{\mathrm{TR}} \gg \tau_{\mathrm{AC}}$. This means that coherent modes "see" a quiescent plastra and can be treated by the usual vlasov theory (in the collisioniess regime that we are concerned with). Only the averaged distribution function is affected by turbulence. In the strong turbulence case, on the other hand, $\tau_{A C} \gg \tau_{T R}$, and coherent modes "see" a turbulent plasma. Thus, in strong turbulence, the dispersion relation for colerent modes is affected by turbulence. The typical effect is to change the ustal resonance function, $\delta(\omega-k v)$, into a function $R(\omega, k, v)$, with finite width. We will assune that we are always in the strong turbslence regime, if turbulence is present at all.

To begin our derivation, we write down the equations of motion for a particle. We let $\theta$ denote the angle around the machine of a particle measured from the position of the "synchronous" particle. We let $\Delta \mathrm{E}$ denote the energy deviation of a particle from the average energy. We let $\mathrm{f}$ denote the revolution frequency of the bunch. We then define

$$
w=\frac{\Delta E}{f}
$$

The equation for $\theta$ then becomes

$$
\frac{d \theta}{d t}=2 \pi \frac{d f}{d E} \Delta E=k_{0} w
$$

where

$$
k_{0}=2 \pi f \frac{d f}{d E}=\frac{2 \pi f^{2}}{E} \alpha
$$


where $\alpha$ is the usual momentum compaction factor and ilepends on the machine parameters. The equation for $w$ is

$$
\frac{d w}{d t}=2 \pi \operatorname{Re} \varepsilon(\theta, t)-\frac{w}{\tau_{r}}+g(t)-\frac{\Omega^{2}}{k_{0}} \theta
$$

where $R$ is the radius of the machine, ${ }^{r_{r}}$ is the radiation damping tine, and $\Omega$ is the synchrotron oscillation frcquency. In Eq. (2.4), the first term is the effect of various electric fields on the particle, and the second term is the effect of radiation damping. The third term, $g(t)$, is a random function with zero mean which represents the effect of quantum noise. The last tem in $(2.4)$ represents the synclurotron oscillations. In this term we include not only the effect of the RF fields, which is given by a synchrotron frequency $\Omega_{0}$, but also the linear effect of the self-fieids on the synchrotron motion. Thus, we explicitly include the equilibrium effects in our calculation. As an equation for $\Omega$, we will use the general expression given by Pellegrini and Sessler. ${ }^{4}$

We let $\psi(\theta, w, t)$ denote the distribution function for particles. Then,

$$
\psi(\theta, w, t+\Delta t)=\int \psi(\theta-\Delta \theta, w-\Delta w, t) P(\theta, w, \Delta \theta, \Delta w, \Delta t, t) d \Delta \theta d \Delta w
$$

where $P(\theta, w, \Delta \theta, \Delta w, \Delta t, t) d \Delta \theta d \Delta w$ is the probability of a change $\Delta \theta, \Delta w$ in time $\Delta t$. If we let $\tau_{r} \rightarrow \infty, g(t) \rightarrow 0, \Omega \rightarrow 0$, then Eqs. (2.2) and (2.4) imply

$$
P(\theta, w, \Delta \theta, \Delta w, \Delta t, t)=\delta\left(\Delta \theta-k_{0} w \Delta t\right) \delta(\Delta w-2 \pi \operatorname{Re} \& \Delta t)
$$

Putting (2.6) into (2.5), expanding to first order in $\Delta t$, then letting $\Delta t \rightarrow 0$, we get

$$
\frac{\partial \psi}{\partial t}+k_{0} w \frac{\partial \psi}{\partial \theta}+2 \pi \operatorname{Re} \varepsilon(\theta, t) \frac{\partial \psi}{\partial w}=0
$$


the usual V1asov equation for unbunched beams.

In order tc take into account turbulence, we will replace $\Gamma$ by an approximate $P$ for a "test" field $\varepsilon_{T}$ plus a turbulent background $\&_{B}$. Dupree $^{8}$ has shown that such a separation leads to a convergent perturbation theory. We will then let $\Delta t \rightarrow \tau$, where $\tau$ is short compared to times of interest, but long enough for particles to "see" significant turbulence.

Let

$$
\theta^{\prime}=\theta-\theta_{0}-k_{0} w \Delta t
$$

and

$$
w^{*}=w-w_{0}-2 \pi \operatorname{Re} \varepsilon_{T} \Delta t+\frac{w}{\tau_{r}} \Delta t+\frac{\Omega^{2}}{k_{0}} \theta \Delta t
$$

where $\theta_{v}, w_{0}$ are the starting values of $\theta, w$, and

$$
\varepsilon(\theta, t)=\varepsilon_{T}(\theta, t)+\varepsilon_{B}(\theta, t)
$$

as discussed above. Then the equations for $\theta^{\prime}, w^{\prime}$ are

$$
\begin{gathered}
\frac{d \theta^{\prime}}{d t}=k_{0} w^{\prime} \\
\frac{d w^{\prime}}{d t}=2 \pi \operatorname{Re} \varepsilon_{B}+g(t)
\end{gathered}
$$

With the assumption that $\varepsilon_{B}(\theta, t)$ represents a turbulent background of waves with random phases, we see, from classical diffusion theory, ${ }^{12}$ that $w^{\prime}$ undergoes a diffusion process with diffusion constant

$$
d=\frac{D}{f^{2}}=(2 \pi R e)^{2} \int_{0}^{\infty} \varepsilon_{B}(\tau) \varepsilon_{B}(t+\tau) d \tau+\frac{D_{Q}}{f^{2}}
$$

where $D_{Q}$ is the usual quantum diffusion constant. ${ }^{13}$ we have assumed no 
correlation between quantum noise and the turbulent background. Now, from Benford and Thompson, ${ }^{11}$ we find that the probability function for $\theta^{\prime}, w^{\prime}$ is

$$
P\left(\theta^{\prime}, w^{\prime}, \Delta t\right)=\frac{e^{-\frac{w^{\prime 2}}{4 d \Delta t}}}{\sqrt{4 \pi d \Delta t}} \frac{e^{-\frac{\left(\theta^{\prime}-k_{0} / 2 k^{\prime} \Delta t\right)^{2}}{\left(\frac{1}{3} d(2 t)^{3}\right)}}}{\sqrt{\frac{1}{3} \pi d(\Delta t)^{3}}}
$$

Using Fqs. $(2.8)$ and $(2.9)$, we find

$$
\begin{aligned}
P\left(\theta, w, \Delta \theta, \Delta w^{2}, \Delta t, t\right)= & \frac{1}{\sqrt{4 \pi d \Delta t}} \exp \left\{-\left[\Delta w-2 \pi R e \varepsilon_{T} \Delta t+\frac{w}{\tau_{r}} \Delta t+\frac{\Omega^{2}}{k_{0}} \theta \Delta t\right]^{2} / 4 \Delta \Delta t\right\} \\
& \frac{1}{\sqrt{\frac{1}{3} \pi d(\Delta t)^{3}}} \times \exp \left\{-\left[\Delta \theta-k_{0} v \Delta t-\frac{\Delta t}{2}\left(k_{0} \Delta w-2 \pi \operatorname{Re} \varepsilon_{T} \Delta t k_{0}\right.\right.\right. \\
+ & \left.\left.\left.k_{0} \frac{w}{\tau_{r}} \Delta t+\Omega^{2} \theta \Delta t\right)\right]^{2} /\left(\frac{1}{3} d(\Delta t)^{3}\right)\right\}
\end{aligned}
$$

Putting (2.15) into (2.5), expanding in $\Delta t$, and keeping only first order terms in $\Delta t$, we find

$$
\frac{\partial \psi}{\partial t}+k_{0} w \frac{\partial \psi}{\partial \theta}+2 \pi \operatorname{Re} \varepsilon_{T} \frac{\partial \psi}{\partial w}-\frac{W}{\tau_{r}} \frac{\partial \psi}{\partial w}-\frac{\Omega^{2} \theta}{k_{0}} \frac{\partial \psi}{\partial w}-\frac{\psi}{T_{r}}=\frac{D}{f^{2}} \frac{\partial^{2} \psi}{\partial w^{2}}
$$

We let

$$
\mathrm{D}=\mathrm{D}_{\mathrm{Q}}+\mathrm{D}_{\mathrm{T}}
$$

where $D_{Q}$ is the quantum diffusion coefficient, and $D_{T}$ is the turbulent diffusion coefficient. Equation (2.16) is our basic equation.

Now, we have assumed that $\Delta t \rightarrow \tau>\tau_{A C}$. In order to neglect higher order terms in $\Delta t$, several assumptions must be made. First, we have to assume that $\tau_{\mathrm{AC}} \ll 1 / \Omega$. Since the modes we shall consider oscillate at multiples of $\Omega$, this is basically a high mode number, or short wavelength, 
assumption. We have also assumed that $D$ is a constant. For $D_{Q}$ this is certainly correct, but for $\mathrm{D}_{\mathrm{T}}$ this requires a broad band turbulence spectrum. This also follows from the assumption of a short autocorrelation time. Various other terms are negligible in the broad band spectrum case. Finally, we must assume $\tau_{A C} \ll 2 d \tau^{2} / w_{\text {RMS }}^{2}$, where $w_{R M S}$ is the root mean square $w$. This condition is equivalent to $\tau_{r} \gg \tau_{A C}$ which will certainly be true in all cases will consider, since $\tau_{r} \gg 1 / \Omega$.

Although our derivation of strong turbulence theory is less general than that of Dupree, ${ }^{8}$ Weinstock, ${ }^{9}$ or Benford and Thompson, ${ }^{11}$ it has the virtue of simplicity; that is, only assuming that particles see a turbulent background allows one to deri,a the usual Fokker-Planck equation. In addition, we will see that we reproduce all of the first order results of the aforementioned authors. Thus, for our purposes, Eq. (2.16) should be an adequate representation of strong turbulence theory.

\section{THE RANDOM PHASE APPROXIMATION}

In deriving $\mathrm{Eq} .(2.13)$, it was necessary to assume random phases for the spectrum of coherent oscillations. This assumption is usually justified by saying that mode-mode coupling causes a rapid exchange of energy between mades, and as a result, the phases seem quite random simply because these are very complicated processes. We believe that some such mechanism takes place in the case we are considering. However, in this section we examine this assunption in more detail.

We consider a particle executing transverse betatron oscillations, moving in a nonlinear longitudinal well, and interacting with short wavelength longitudinal waves of definite phases. We will show, using a criterion due to Chirikov, ${ }^{14}$ that such a particle has a "stochastic". 
i.e., randon, trajectory. Since the particle interacts with the wave, this randomess results in a stochastic transfer of energy to and from the wave. Thus, the phase of the wave becomes random. Through mode-mode coupling, wijch we assume exists, this randomess is fed down into the lower order modes. As a result, it is justified to assume random phasas of all waves.

In our model, we assurne that the magnitude of the short wavelength modes is proportional to the average particle density at that transverse position; i.e., we assume that it is a Gaussian in the transverse coordinate, $x$. Thus,

$$
E=\sum_{m \neq 0} E_{m} e^{j m \theta-\frac{x^{2}}{2 \sigma^{2}}}
$$

where $\sigma$ is the root mean square transverse displacement. Our equations of motion become

$$
\begin{aligned}
& \dot{\theta}=k_{0} w \\
& \dot{w}=2 \pi \operatorname{Re} \varepsilon_{0}(\theta, t)+2 \pi \operatorname{Re} \sum_{m \neq 0} E_{m} e^{i m \theta-\frac{x^{2}}{2 \sigma^{2}}} \\
& \ddot{x}=-\omega_{\beta}^{2} x+\frac{2 \pi i \operatorname{Re} x}{\sigma^{2}} \sum_{m \neq 0} \frac{E_{m}}{m} e^{i m \theta-\frac{x}{2 \sigma^{2}}}
\end{aligned}
$$

where $\omega_{B}$ is the betatron oscillation frequency, and $\delta_{0}$ is the RF field.

In order to show stochasticity of the particle trajectories, we will use the Chirikov criterion. ${ }^{14}$ To apply this criterion one first solves the lowest order equations; in our case, the equations with $E_{m}=0$. An oscillatory motion must be assuncd to be nonlinear; we will assune the synchrotron motion to be nonlinear. One then puts the lowest order solution into the perturbing term and treats it as a time-dependent 
perturbation. Clearly this can't be assumed to give the solution for all time, since energy is conserved, but is adequate for deriving the stochasticity threshold. Since the unperturbed motion is nonlinear, its frequency depends on amplitude. Typically, the perturbing terms will have many frequencies presen:, and at any oscillation amplitude, many of the perturbing terms will be is resonance. Isolating any one resonance, one sees that it gives rise to a resonance region of finite width, since the unperturbed motion is assumed to be nonlinear. We call the width in amplitude of this resonance $\delta_{W}$. The distance between resonances is called $\Delta_{R}$. When resonances overlars, i.e. when $\delta_{W} \geq \Delta_{R}$, the assumption is that stochasticity sets in. The rough idea is that particles can move from resonance to resonance in a random fashion. Chirikov ${ }^{14}$ has investigated this criterion with numerical simulation and found it to be remarkably accurate.

To apply this criterion in our case we write down the lowest order equations. From $(3.2),(3.3)$, and $(3.4)$, these are

$$
\begin{aligned}
& \ddot{x}=-w_{\beta}^{2} x \\
& \ddot{\theta}=2 \pi \operatorname{Re} \&_{0}(\theta, t)
\end{aligned}
$$

The solution to $(3.5)$ is clearly

$$
x=x_{0} \sin \left(\omega_{\beta} t+y\right)
$$

where $x_{0}$ is the amplitude and $\gamma$ the initial phase. The solution of (3.6) is, in lowest order,

$$
\theta=\theta_{0} \cos \Omega_{1} t+\frac{k_{0} w_{0}}{\Omega} \sin \Omega_{1} t
$$

where 


$$
\Omega_{1}=\Omega\left[1-\frac{3 \varepsilon}{8}\left(0_{0}^{2}+\frac{k_{0}^{2} w_{0}^{2}}{\Omega^{2}}\right)\right]
$$

Equations (3.8) and (3.9) were obtaincd by assuning a cubic nonlinearity with coefficicnt $E$ and using the usual first order solution. ${ }^{15}$ Equation (3.9) shows the amplitude dependence of the frequency of oscillation of ๑. Let

$$
\begin{aligned}
& I=\frac{k_{0} k_{0}^{2}}{2 \Omega}+\frac{\Omega \theta^{2}}{2 k_{0}} \\
& \Omega_{1}(I)=\Omega\left(1-\frac{3 \varepsilon k_{0}}{4 \Omega} 1\right)
\end{aligned}
$$

The lowest order Haniltonian is then

$$
H_{0}=\int^{I} \Omega\left(I^{\prime}\right) d^{\prime}
$$

Using the solution (3.7) in the perturbation term of (3.3), the next order Haniltonian for longitudinal motion becomes

$$
H=H_{0}+2 \pi \operatorname{Re} i \sum_{m \neq 0} \frac{E_{m}}{m} e^{i m \sqrt{2 k_{0} I / \Omega} \sin \psi} \times \exp \left\{\frac{-x_{0}^{2}}{40^{2}}+\frac{x_{0}^{2} \cos \left[2\left(\omega_{B} t+\gamma\right)\right]}{40^{2}}\right\}
$$

where $\psi$ is the angle variable canonically conjugatc to I. We now use

$$
\begin{aligned}
& e^{i m \sqrt{2 k_{0} I / \Omega \sin \psi}=\sum_{n=-\infty}^{\infty}(-1)^{n} J_{n}\left(m \sqrt{2 k_{0} I / \Omega}\right) e^{-i n \psi}} \\
& \exp \left\{\frac{x_{0}^{2}}{4 \sigma^{2}} \cos \left[2\left(\omega_{B} t+\gamma\right)\right]\right\}=\sum_{R=-\infty}^{\infty} I_{\ell}\left(\frac{x_{0}^{2}}{4 \sigma^{2}}\right) e^{i \ell\left[2\left(\omega_{B} t+\gamma\right)\right]}
\end{aligned}
$$

where $J_{n}$ and $J_{\ell}$ are the usunl Besse! functions. Putting (3.14) and (3.15) jnto (3.1.3) we rind 


$$
\begin{aligned}
H & =H_{0}+2 \pi R e i \exp \left\{\frac{-x_{0}^{2}}{4 \sigma^{2}}\right\} \times \sum_{m \neq 0} \sum_{n=-\infty}^{\infty} \sum_{\ell=-\infty}^{\infty} \frac{E_{m}}{m} \\
& \times(-1)^{n} J_{n}\left(m \sqrt{2 k_{0} I / \Omega}\right) I_{\ell}\left(\frac{x_{0}^{2}}{4 \sigma^{2}}\right) e^{i \ell\left[2\left(\omega_{B} t+\gamma\right)\right]-i n \psi}
\end{aligned}
$$

Since we have

$$
\psi \sim \Omega_{1} t
$$

we see that resonance occurs when

$$
2 \ell \omega_{\beta} \simeq \mathrm{n} \Omega_{1}
$$

Since $\omega_{B} \gg \Omega$, we expect that $\ell= \pm 1$ will be the dominant terms. For each $n$, there is a value of $I$, call it $I_{n}$, for which

$$
\pm 2 \omega_{B}=n \Omega_{1}\left(I_{n}\right)
$$

For values of $I$ near $I_{n}$, only one term in the sum over $n$ will contribute. Selecting this term and letting

$$
\begin{aligned}
& \bar{\psi}=n \psi-2 \omega_{\beta} t \\
& \bar{I}=\frac{1}{n}\left(I-I_{n}\right)
\end{aligned}
$$

the Hamiltonian is approximately

$$
H=\left.n^{2} \frac{d \Omega_{1}}{d I}\right|_{I_{n}} \frac{\bar{I}^{2}}{2}+U_{n} \cos (\bar{\psi}-2 \gamma)
$$

where

$$
U_{n}=4 \pi R e i \exp \left(\frac{-x_{0}^{2}}{4 \sigma^{2}}\right)(-1)^{n} I_{1}\left(\frac{x_{0}^{2}}{4 \sigma^{2}}\right) \sum_{m \neq 0} \frac{E_{m}}{m} J_{n}\left(m \sqrt{2 k_{0} I_{n} / \Omega}\right)
$$

Equation (3.22) is the usual Hamiltonian for a pendulum, which has a closed region of bounded trajectories with width in $I$ of 


$$
\delta_{W}=8 \sqrt{\left|\frac{U_{n}}{3 \varepsilon k_{0} n^{2}}\right|}
$$

where we used

$$
\frac{\mathrm{d} \Omega_{1}}{\mathrm{dI}}=-\frac{3 \varepsilon \mathrm{k}_{0}}{4}
$$

Now, from (3.11) and (3.19), we have

$$
1-\frac{3 \varepsilon k_{0}}{4 \Omega} I_{n}=\frac{2 \omega_{B}}{n \Omega}
$$

Thus,

$$
I_{n}-I_{n+1}=\frac{8 \omega_{B}}{-3 \varepsilon k_{0}}\left(\frac{1}{n} \cdot \frac{1}{n+1}\right) \approx \frac{8 \omega_{B}}{-3 k_{0} \varepsilon n^{2}}
$$

Using (3.27) and (3.21) we find

$$
\Delta_{R}=\left|\frac{8 \omega_{B}}{3 \varepsilon k_{0} n^{3}}\right|
$$

thus the Chirikov criterion becomes

$$
1 \leq \frac{\delta_{W}}{\Delta_{R}}=n^{2} \frac{\sqrt{\left|3 E k_{0} U_{n}\right|}}{\omega_{B}}
$$

Now, 16

$$
2 \pi R E_{m}=-Z_{m} \lambda_{m} e \beta c
$$

where $\lambda_{m}$ is the $m^{\text {th }}$ Fourier component of charge density, $\beta=v / c=1$ is the velocity in units of $c$, the speed of light. $z_{m}$ is an impedance which depends on the beam surroundings. We want to estimate the size of $\lambda_{m} / \lambda_{2}$ for which (3.29) is satisfied. Faking $x_{0}=\sigma$, and using (3.30) in (3.23), we get 


$$
\left|U_{n}\right|=.2 \beta c\left\langle\frac{Z_{m}}{m}\right\rangle\left\langle\frac{\lambda_{m}}{\lambda_{0}}\right\rangle e^{2} \lambda_{0} \sum_{j \neq 0}^{1} J_{n}\left(m \sqrt{2 k_{0} I} / \Omega\right)
$$

where $\left(z_{\mathrm{m}} / \mathrm{m}\right)$ is assumed roughly independent of $\mathrm{m}$, in agreement with our fits to the data, and $\left\langle\lambda_{\mathrm{m}} / \lambda_{0}\right\rangle$ is an average size of the coherent modes. The prime on the sum indicates that phases should be taken into account. Since

$$
\sqrt{2 \mathrm{k}_{0} \mathrm{I}_{\mathrm{n}} / \Omega} \Rightarrow \theta_{\mathrm{RMS}}
$$

we conclude that $m \sqrt{2 k_{0} I_{n} / \bar{\Omega}}>1$. Thus,

$$
J_{n}\left(m \sqrt{2 k_{0} I / n}\right) \propto \frac{1}{\sqrt{2 \pi m \theta}{ }^{/ R M S}}
$$

Since about $\sqrt{\mathrm{n}}$ terms should add coherently, we find

$$
\sum_{m \neq 0}^{\prime} J_{n}\left(m \sqrt{2 k_{0} I / \Omega}\right) \sim \frac{1}{\sqrt{\theta_{\text {RMS }}}}
$$

Thus,

$$
\left|u_{n}\right|=.2\left\langle\frac{z}{m}\right\rangle\left\langle\frac{\lambda_{m}}{\lambda_{0}}\right\rangle e^{2} \frac{\lambda_{0}}{\sqrt{\theta_{\mathrm{RMS}}}} B c
$$

Let us assume

$$
n=\frac{2 \omega_{B}}{\Omega}
$$

and use machine parameters relevant to SPEAR I, namely

$$
\begin{aligned}
& \mathbf{f}=1.28 \times 10^{6} \mathrm{sec}^{-1} \\
& \lambda_{0}=\frac{\mathrm{N}}{2 \pi \mathrm{R}}=\frac{5 \times 10^{10}}{2 \pi(37.3 \mathrm{~m})}=2.13 \times 10^{6} \mathrm{~cm}^{-1} \\
& B=1
\end{aligned}
$$




$$
\begin{aligned}
\theta_{R M S} & \cong 2.68 \times 10^{-3} \\
E & =1.5 \mathrm{GeV} \\
\left|k_{0}\right| & =\frac{2 \pi f^{2}}{E} a, \quad a=.041 \\
\omega_{B} & \cong 2.66 \times 10^{7} \mathrm{sec}^{-1} \\
\Omega & \cong 8 \times 10^{3} \mathrm{sec}^{-1} \\
\varepsilon & \cong 270 .
\end{aligned}
$$

We will also assume that

$$
\frac{z_{m}}{m}=1 \mathrm{ohm}
$$

Putting (3.35) into (3.29) and using these values, we get

$$
\left\langle\frac{\lambda_{m}}{\lambda_{0}}\right\rangle \geq 4 \times 10^{-5}
$$

Since we have included one term in the sum over $\ell$ in (3.16), the actual stochasticity boundary is probably somewhat smaller. In any case, we conclude that even very small amplitude waves will give rise to stochastic particle trajectories.

The magnitude of the impedance that we have assumed is reasonable and is about the same as that required to fit the bunch lengthening data. Thus, although some of the approximations we made were very crule, in particular Eqs. (3.31) and (3.34), our result can be taken as an indication that particle trajectories are stochastic, and that the random phase approximation is justified. 
IV. CONVERSION TO AN INTEGRAL EQUATION

In this section, we begin the derivation of the dispersion relations from Eq. (2.16). We assune small perturbations about an equilibrium state and linearize in these small terms. The effect of nonlinear terms, namely m. . Illudc cuupling, will be discusscd, : .ul calculated, wisen we eximine the experimental data.

Equation (2.16) has a steady state solution given by

$$
\Psi_{0}(\theta, w)=M \exp \left\{-\frac{f^{2}}{2 D \tau_{r}}\left(w^{2}+\frac{\Omega^{2} \theta^{2}}{k_{0}^{2}}\right)\right\}
$$

where $M$ is a normalization constant. We let

$$
\psi=\psi_{0}+\psi_{1}
$$

and, keeping only terms linear in $\psi_{1}$, from (2.15) we get

$$
\frac{\partial \psi_{1}}{\partial t}+k_{0} w \frac{\partial \psi_{1}}{\partial \theta}-\left(\frac{w}{\tau_{r}}+\frac{\Omega^{2} \theta}{k_{0}}\right) \frac{\partial \psi_{1}}{\partial w}-\frac{\psi_{l}}{\tau_{r}}=\frac{D}{f^{2}} \frac{\partial^{2} \psi_{1}}{\partial w^{2}}-2 \pi \operatorname{Re} \& \frac{\partial \psi_{0}}{\partial w}
$$

In order to solve Eq. (4.3) for $\psi_{1}$, we consider the equation

$$
\frac{\partial h}{\partial t}+k_{0} w \frac{\partial h}{\partial \theta}-\left(\frac{w}{\tau_{r}}+\frac{\Omega^{2} \theta}{k_{0}}\right) \frac{\partial h}{\partial w}-\frac{h}{\tau_{r}}=\frac{D}{f^{2}} \frac{\partial^{2} h}{\partial w^{2}}
$$

In terms of the solution to Eq. $(4.4)$, with the initial condition

$$
h\left(\theta, w, t_{0} ; \theta_{0}, w_{0}, t_{0}\right)=\delta\left(\theta-\theta_{0}\right) \delta\left(w-w_{0}\right)
$$

the solution of Eq. (4.3) satisfies

$$
\begin{aligned}
\psi_{1}(\theta, w, t) & =\int h\left(\theta, w, t ; \theta_{0}, w_{0}, 0\right) \psi_{1}\left(\theta_{0}, w_{0}, 0\right) d \theta_{0} d w_{0} \\
& -\left.2 \pi \operatorname{Re} \int_{0}^{t} d \tau \int d \theta_{0} d w_{0} h\left(\theta, w, t ; \theta_{0}, w_{0}, \tau\right)\left(\varepsilon\left(\psi_{1}\right) \frac{\partial \psi_{0}}{\partial w}\right)\right|_{\theta_{0}, w_{0}, \tau}(4.6) .
\end{aligned}
$$


We have written $g=\varepsilon\left(\psi_{2}\right)$ to indicate its dependence on $\psi_{1}$. We have taken $t_{0}=0$. The method of solution that we are using is a generalization of the method of integration over characteristics of the unperturhal orbits. If there were no diffusion, there would be exact unperturbed orbits. When a diffusion term is present, we are taking a weighted average over an ensemble of unperturbed orbits. In any case, Eq. (4.6) is an exact consequence of $\mathrm{Eq} \cdot(4 \cdot 3)$.

The solution of Eq. (4.4) which satisfies Eq. (4.5) is known. ${ }^{12}$ . ar convenience, we present a derivation of it in Appendix A.

Since Eq. (4.4) is homogeneous in time, the solution, h, satisfics

$$
h\left(\theta, w, t ; \theta_{0}, w_{0}, t_{0}\right)=h\left(\theta, w ; \theta_{0}, w_{0} ; t-t_{0}\right)
$$

Thus, we can Laplace transform Eq. $(4,6)$ in time and use the convolution theorem for Laplace transforms to obtain

$$
\begin{aligned}
\widetilde{\psi}_{1}(\theta, w, s)= & \int \tilde{h}\left(\theta, w ; \theta_{0}, w_{0}, s\right) \psi_{1}\left(\theta_{0}, w_{0}, 0\right) d \theta_{0} d w_{0} \\
& -\left.2 \pi \operatorname{Re} \int d \theta_{0} d w_{0} \tilde{h}\left(\theta, w ; \theta_{0}, w_{0}, s\right)\left(\varepsilon\left(\tilde{\psi}_{1}\right) \frac{\partial \psi_{0}}{\partial w}\right)\right|_{\theta_{0}, w_{0}, s}
\end{aligned}
$$

where we have assumed that $\varepsilon$ is a linear operator on $\psi_{1}$. Let

$$
F(\theta, w, s)=\int \tilde{h}\left(\theta, w ; \theta_{0}, w_{0}, s\right) \psi_{1}\left(\theta_{0}, w_{0}, 0\right) d \theta_{0} d w_{0}
$$

which is the initial value term. From Eq. $(4,1)$, we have

$$
\dot{\psi}_{0}\left(\theta_{0}, w_{0}\right)=M P\left(\theta_{0}\right) W\left(w_{0}\right)
$$

Let

$$
L\left(\theta, w, \theta_{0}, s\right)=\int d w_{0} \tilde{h}\left(\theta, w / \theta_{0}, w_{0}, s\right) \frac{d w\left(w_{0}\right)}{d w}
$$

Equation (4.8) is now 


$$
\tilde{\psi}_{1}(\theta, w, s) \Rightarrow F(\theta, w, s)-\left.2 \pi \operatorname{Re} M \int d \theta_{0} L\left(\theta, w, \theta_{0}, s\right) P\left(\theta_{0}\right) \&\left(\tilde{\psi}_{1}\right)\right|_{\theta_{0}, s}
$$

We have used the fact that 8 is a function only of $\theta_{0}$ and not of $w_{0}$. Now we use

$$
\mathscr{E}\left(\theta_{0}, t\right)=\frac{\mathrm{fR}}{B C} \int d \theta^{\prime} \lambda\left(\theta^{\prime}, \mathrm{t}\right) \mathrm{G}\left(R\left(\theta^{\prime}-\theta_{0}\right)\right)
$$

where $\lambda\left(\theta^{\prime}, t\right)$, the charge density per unit length, is given by

$$
\lambda\left(\theta^{\prime}, t\right)=\frac{e}{R} \int \psi\left(\theta^{\prime}, w, t\right) d w
$$

Equation (4.13; is a consequence of the linearity of Maxwe11's equations. In particular, it is exactly the same form used by Pellegrini and Sessler. ${ }^{4}$ $G$ is a Green's function which includes the effect of the beam surroundings. In principle $\mathrm{G}$ is calculable once the details of the particular machine are known. In fact $G$ has been calculated for various types of structures. $^{4}$ In practice, however, $G$ is very poorly known. Fortunately our results are relatively insensitive to the details of this function. Putting Eqs. (4.13) and (4.14) into Eq. (4.12), we obtain

$$
\begin{aligned}
\tilde{\psi}_{1}(\theta, w, s) & =F(\theta, w, s)-2 \pi \operatorname{Re} M \int d \theta_{0} L\left(\theta, w, \theta_{0}, s\right) P\left(\theta_{0}\right)\left(\frac{f e}{B c}\right) \\
& \times \int d \theta^{\prime} G\left(R\left(\theta^{\prime}-\theta_{0}\right)\right) \int \widetilde{\psi}_{1}\left(\theta^{\prime}, w, s\right) d w
\end{aligned}
$$

Equation (4.15) is a linear integral equation for $\tilde{\psi}$, from which we will obtain a dispersion relation. 


\section{UNIFORM BEAMS}

In this section we obtain the dispersion relation for a uniform, i.e., unbunched beam. Although the complete solution has no obvious applications, the special case in which radiation damping is absent describes turbulent, uniform, proton beams. Our derivation also reproduces the previous result of Sessler. ${ }^{7}$

The uniform beam case results when we take the 1 imit $\Omega \rightarrow 0$. In this $1 \mathrm{imit}$, Eq. (4.4) is translationally invariant in $\theta$, and thus

$$
h\left(\theta, w ; \theta_{0}, w_{0}, t-t_{0}\right)=h\left(\theta-\theta_{0}, w, w_{0}, t-t_{0}\right)
$$

Thus, $\mathrm{L}$ in Eq. (4.11) depends only on $\theta-\theta_{0}$. Also, in (4.10),

$$
P\left(\theta_{0}\right)=1
$$

Thus we can Fourier transform Eq. $(4,15)$ in $\theta$, and use the convolution theorem for Fourier transforms. We get

$$
\widetilde{\Psi}_{2}(p, w, s)=\tilde{F}(p, w, s)-(2 \pi)^{2} \operatorname{Re} M\left(\frac{f e}{B c}\right) \tilde{L}(p, w, s)(\overbrace{\left.\int d \theta^{\prime} G \int \tilde{\psi}_{1} d w\right)}
$$

In Eq. (5.3) we again use the convolution theorem for the last term along with the fact that

$$
\tilde{G}(p)=\frac{B c}{2 \pi R} Z\left(-\frac{p B c}{R}\right)
$$

In Eq. (5.4), $Z$ is the impedance function. The relation between this impedance fumction and that defined by Eq. (3.30) is

$$
z\left(-p w_{0}\right)=-z p
$$

where

$$
\omega_{0}=\frac{B C}{R}=2 \pi f
$$


Equation (5.3) becomes

$$
\tilde{\psi}_{1}(p, w, s)=\tilde{F}(p, w, s)-\frac{(2 \pi)^{3} \operatorname{Re}^{2} M f^{2}}{B c} \tilde{L}(p, w, s) Z\left(-p \omega_{0}\right) \int \tilde{\psi}_{1} d w
$$

If we define

$$
\begin{aligned}
& \phi(p, s)=\int d w \tilde{\psi}_{1}(p, w, s) \\
& \bar{F}(p, s)=\int d w \tilde{F}(p, w, s) \\
& \bar{L}(p, s)=\int d w \tilde{L}(p, w, s)
\end{aligned}
$$

then from Eq. (5.7) we get

$$
\phi(p, s)=\bar{F}(p, s)-\frac{(2 \pi)^{3} \operatorname{Re}^{2} M f^{2}}{B c} \bar{L}(p, s) Z\left(-p \omega_{0}\right) \phi(p, s)
$$

We recall that $\overline{\mathrm{F}}(\mathrm{p}, \mathrm{s})$ is an initial value tem.

In this case the normalization constant is

$$
M=\frac{f N}{\sqrt{2 \pi D \tau}}
$$

where $\mathrm{N}$ is the total number of particles in the heam. Thus from Eq. (5.11) we find

$$
\phi(p, s)=\frac{\bar{F}(p, s)}{1+\frac{(2 \pi)^{3} \operatorname{Re}^{2} f^{3} N Z\left(-p_{\omega_{0}}\right)}{B c \sqrt{2 \pi D \tau} r} \bar{L}(p, s)}
$$

The zeros of the denominator of this expression give, as usual, the dispersion relation. In Appendix $B$ the function $\bar{L}(p, s)$ is calculated. The result is 


$$
\vec{L}(p, s)=i p k_{0} \sqrt{\frac{D \tau^{5}}{2 \pi r^{2}}} \int_{0}^{\infty} d x x \exp \{-v x+(s \tau r-1+v) \ln (x+1)\}
$$

In Eq. (5.14) we have defined

$$
v=D\left(\frac{p k_{0}}{I}\right)^{2} \tau_{r}^{3}
$$

thus the dispersion relation is

$$
1+N e^{2}\left\{z\left(-p \omega_{0}\right) i p k_{0} \tau^{2} T \int_{0}^{\infty} d x x \operatorname{cxp}\{-v x+(s-1+v) \ln (1+x)\}=0\right.
$$

In Appendix C, we present another derivation of Eq. (5.16). We show that this result is identienl with that obtained by Sessler, ? even though Sessler magipulated divergent integrals to obtain his result.

\section{INTEGRAL EQUATION FOR BIRACHED BEAMS}

In this section we will derive an integral equation for busched beams. Since we are concerned with bunched beans whose length is much smaller than the machine circumference, we extend the integrations in angle in Eq. (4.15) to the interval $[-\infty, \infty]$. Though not exact, the rapid fall-off in angle of $\psi_{0}(\theta, w)$ nakes this an excellent approximation.

The usual method ${ }^{17,18}$ of solving Eq. (2.16), when damping and diffusion are absent, is to transform to action-angle variables

$$
\begin{aligned}
& J=w^{2}+\frac{\Omega^{2} \theta^{2}}{k_{0}^{2}} \\
& \phi=\cos ^{-1} \frac{w}{\sqrt{J}} .
\end{aligned}
$$


The cquation is then fouriet erassformed in $\$$. In this approach the impedance function enters in a very complitated fashion, and only special iypes of impelance functions can be trated. The presence of the diffusion term makes the transformution to ation-angle variables incifetive. Instead, we mist work with the Fourier transform in the physical angle $:$. As a result the ingedaxe functions enters in a relatively si:ale musrer.

Since we are only interested in the response of the bean as a funcrion of angle, let us define

$$
\begin{aligned}
\zeta(\theta, s) & =\int d w \tilde{\psi}_{2}(\theta, w, s) \\
\sigma(\theta, s) & =\int d w F(\theta, w, s) \\
K(\theta, \theta, s) & =\int d w I .\left(\theta, w, \theta_{0}, s\right)
\end{aligned}
$$

Using these definitions, Eq. (4.15) implies

$$
\xi(\theta, s)=a(\theta, s) \cdot \frac{2 \pi R c^{2} M f}{B C} \int d \theta_{0} K\left(\theta, \theta_{0}, 5\right) P\left(\theta_{0}\right) \int d \theta^{\prime} G\left(R\left(\theta^{\prime}-\theta_{0}\right)\right) \xi\left(\theta^{\prime}, s\right)
$$

Let us fouricr transform in $\theta$.

$$
\tilde{\xi}(p, s)=\tilde{\sigma}(p, s)-\frac{2 \pi R^{2} M f}{B c} \int d \theta_{0} \tilde{K}\left(p, \theta_{0}, s\right) P\left(\theta_{0}\right) \int d \theta^{\prime} G\left(R\left(\theta^{\prime}-\theta_{0}\right)\right) \xi\left(\theta^{\circ}, s\right)
$$

Recall that

$$
\int d \theta_{0} f\left(\theta_{0}\right)=2 \pi \tilde{f}(0)
$$

The last term in Eq. (6.5) is precisely of this form. Since the integrand of the last term in Eq. (6.5) is a product, its Fourier transform is a convolution, i.c. 


$$
\left.\tilde{K}\left(p, \theta_{0}, s\right) P^{\prime}\left(\theta_{0}\right) \int d \theta^{\prime} G\left(R\left(\theta^{\prime}-\theta_{0}\right)\right) \xi\left(\theta^{\prime}, s\right)\right|_{p, r, s}=
$$

Using Eq. (5.4) and the convolution theorem, we see that

$$
\left.\int d \theta^{\prime} G\left(R\left(\theta^{\prime}-\theta_{0}\right)\right) \xi\left(\theta^{\prime}, s\right)\right|_{p, r_{1}, s}=\frac{B C}{R} Z\left(-r_{1} \omega_{0}\right) \tilde{\xi}\left(r_{1}, s\right)
$$

Let us define

$$
I\left(p, r_{1}, s\right)=\left.\overbrace{\tilde{K}\left(p, \theta_{0}, s\right) P\left(\theta_{0}\right)}\right|_{p,-r_{1}, s}
$$

Equations (6.6), (6.7), (6.8), and (6.9) imply that Eq. $(6.5)$ is equivalent to

$$
\tilde{\xi}(p, s)=\tilde{\sigma}(p, s)-(2 \pi)^{2} e^{2} M f \int d r_{1} I\left(p, r_{1}, s\right) z\left(-r_{1} \omega_{0}\right) \tilde{\xi}\left(r_{1}, s\right)
$$

Equation $(6.10)$ is the basic integral equation for bunched beams. $\tilde{\sigma}(p, s)$ is an initial value term, and we want to solve for $\tilde{\xi}(p, s)$. For bunched beams the normalization constant is given by

$$
M=\frac{f^{2} \Omega N}{2 \pi D \tau_{r}\left|k_{0}\right|}
$$

where $N$ is the number of particles in the beam. From Eqs. (4.11), (6.3), and (6.9), we get

$$
\begin{aligned}
I\left(p, r_{1}, s\right) & =\frac{1}{(2 \pi)^{2}} \int_{0}^{\infty} d t e^{-s t} \int d \theta_{0} d \theta d w d w_{0} e^{i r_{1} \theta_{0}-\mathbf{i p} \theta} \times \\
& \times P\left(\theta_{0}\right) \frac{d w\left(w_{0}\right)}{d w} h\left(\theta, w, \theta_{0}, w_{0}, t\right)
\end{aligned}
$$


In Eq. $(6.10)$, we see that the contribution of $\tilde{\xi}\left(r_{1}, s\right)$ is weighted by the impedance at the appropriate frequency, $Z\left(-r_{1} \omega_{0}\right)$; a fact which agrees with our physical intuition. The contribution of $\tilde{\xi}\left(r_{1}, s\right)$ is also weighted by the function $I\left(p, r_{1}, s\right)$, which depends on the unperturbed distribution function and on the solution to Eq. (4.4). Fortunately, $I\left(p, r_{1}, s\right)$ can be calculated in the cases of interest. In fact, viten damping and diffusion are absent, the calculation of $I\left(p, r_{1}, s\right)$ is rather simple, as we shall show in the next section. 
VII. BLNGIEI) PROTON BEMLS WTTOUT TRRULENCl:

In this section we obtain the disjersion relation for proton beams from Fq. (6.10). For proton heams, radiation dang̣ing̣ and quantue diffusion are absent. If turbulent diffusion is presenc, the situstion is rather complicated. Because radiat ion damping is absent, particles will diffuse to such large symchrotron anpl itudes that they are lost, or to such large amplitudes that Landau damping due to nonlinnarities will damp the coherent modes. The latter case requires an extensive study of Landau damping. Though we will give an approximate analysis of Landau damping, it will not be exact enough to consider this case. In any event, it seens desirable to avoid, if possible, the onset of turbulence in proton beams. Thus, one should require, if possible, the stability of coherent oscillations. We will assume that turbulent diffusion is absent.

The dispersion relation for proton beams has been considered previously. ${ }^{17,18}$ Sacherer, ${ }^{17}$ in particular, has given a description of the modes in special cases. However, these treatments have been hindered by two problems. First, it, was difficult to include Landau damping in any simple way; only in special cases could Landau daimping be included. Secondly, as commented on previously, the impedance function entered into the calculations in a very complicated way, and as a result, only special impedance functions could be used. In our derivation, the impedance enters in a simple way, and general impedance functions can be used. In addition, although we cannot give an exact treatment of Landau damping, we do give an approximate analysis for all cases. The exact treatment of Landau darping, in our approach, reduces to the analysis of a complicated integral. Furthermore, the integral is the same for all cases if the same distribution 
function is assumed. Thus, at worst, a detailled treatment of Landau damping requires only a numerical investigation of this one integral.

Since we have ascimed no radiation damping and no diffusion, the solution of liq. $\{4.4\}$ which satisfies $(4.5)$ is

$$
h\left(\theta, w, t ; \theta_{0}, w_{0}, 0\right)=\delta\left(\theta-A\left(\theta_{0}, w_{0}, t\right)\right) \delta\left(w-B\left(\theta_{0}, w_{0}, t\right)\right)
$$

where

$$
\begin{aligned}
& A\left(\theta_{0}, w_{0}, t\right)=\theta_{0} \cos \Omega t+\frac{k_{0} w_{0}}{\Omega} \sin \Omega t \\
& B\left(\theta_{0}, w_{0}, t\right)=w_{0} \cos \Omega t+\frac{\Omega \theta_{0}}{k_{0}} \sin \Omega t
\end{aligned}
$$

Using Eqs. (7.1) and (7.2) in Eq. (6.12), we easily find

$$
\begin{aligned}
l\left(p, r_{1}, s\right) & =\frac{1}{(2 \pi)^{2}} \int_{0}^{\infty} d t e^{-s t} \int d \theta_{0} d w_{0} P\left(\theta_{0}\right) \frac{d w\left(w_{0}\right)}{d w} \\
& \times \exp \left\{i r_{1} \theta_{0}-i p\left(\theta_{0} \cos \Omega t+\frac{k_{0} w_{0}}{\Omega} \sin \Omega t\right)\right\}
\end{aligned}
$$

Since $P\left(\theta_{0}\right)$ and $W\left(w_{0}\right)$ are Gaussians, given by Eqs. (4.1) and (4.10), the integrals over $\theta_{0}$ and $w_{0}$ in (7.4) are easily done. The result is

$$
\begin{aligned}
I\left(p, r_{1}, s\right) & =\frac{D \tau_{r}\left|k_{0}\right| i p k_{e}}{2 f^{2} \Omega^{2}} \exp \left\{-\frac{D \tau_{r} k_{0}^{2}\left(p^{2}+r_{1}^{2}\right)}{2 f^{2} \Omega^{2}}\right\} \\
& \times \int_{0}^{\infty} d t e^{-s t} \sin \Omega t \exp \left\{\frac{D \tau_{r} k_{0}^{2} r_{1} p}{f^{2} \Omega^{2}} \cos \Omega t\right\}
\end{aligned}
$$

Note that although we have taken the limit $D \rightarrow 0, \tau_{r}+\infty$, we have kept $D \tau_{r}$, the energy spread of the beam, fixed. Thus only the combination, $D \tau_{r}$, appears in Eq. (7.5) .

Now let us use the fact that ${ }^{19}$ 


$$
\exp \left\{\frac{D \tau_{r} k_{0}^{2} r_{1} p}{f^{2} \Omega^{2}} \cos s t\right\}=I_{0}\left(\frac{D r_{r} k_{0}^{2} r_{1} P}{f^{2} \Omega^{2}}\right)+2 \sum_{k=1}^{\infty} I_{k}\left(\frac{D r_{r} k_{0}^{2} r_{1} p}{f^{2} \Omega^{2}}\right) \cos k: t
$$

where $I_{k}$ denote the usual Bessel functions. Futting Eq. (7,6) into Bq.

(7.5) and performing the integration in $t$, se get

$$
\begin{aligned}
I\left(p, r_{1}, s\right) & =\frac{D \tau_{r}\left|k_{0}\right| i p k_{0}}{2 f^{2} \Omega^{2}} \exp \left\{\frac{-D r_{r} k_{0}^{2}\left(p^{2}+r_{1}^{2}\right)}{2 f^{2} \Omega^{2}}\right\} \\
& \times\left\{I_{0}\left(\frac{D \tau_{r} k_{0}^{2} r_{1} p}{f^{2} \Omega^{2}}\right) \frac{\Omega}{s^{2}+\Omega^{2}}+\sum_{k=1}^{\infty} i_{k}\left(\frac{D r_{r} k_{0}^{2} r_{1} p}{f^{2} \Omega^{2}}\right)\right. \\
& \left.\times\left[\frac{\Omega(k+1)}{s^{2}+\Omega^{2}(k+1)^{2}}-\frac{\Omega(k-1)}{s^{2}+\Omega^{2}(k-1)}\right]\right\}
\end{aligned}
$$

We note that $I\left(p, r_{1}, s\right)$ is large when $s=$ in , with $n$ a positive or nogative integer. Thus, coherent oscillations occur at multiples of the synclirotron frequency, as was to be expected. When $s \approx \mathrm{i} \Omega$, we shall keep only the largest terms; and hence

$$
\begin{aligned}
I\left(p, r_{1}, s\right) & =\frac{D \tau_{r} l k_{0} l i p k_{0}}{2 f^{2} \Omega^{2}} \exp \left\{\frac{-D \tau_{r} k_{0}^{2}\left(p^{2}+r_{1}^{2}\right)}{2 f^{2} \Omega^{2}}\right\} \\
& \times \frac{\Omega n}{s^{2}+n^{2} \Omega^{2}}\left[I_{n-1}\left(\frac{D \tau_{r} k_{0}^{2} r, p}{f^{2} \Omega^{2}}\right)-I_{n+1}\left(\frac{D r_{r} k_{0}^{2} r_{2} p}{f^{2} \Omega^{2}}\right)\right]
\end{aligned}
$$

We now use the identity, ${ }^{19}$

$$
I_{n-1}(Z)-I_{n+1}(Z)=\frac{2 n}{Z} I_{n}(Z)
$$

to find 


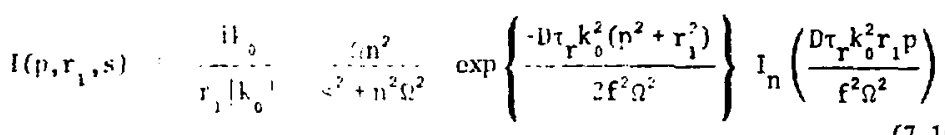

We have not, up to this point, included Landau damping in the calculation since we have assimed linear binding forces. Because particles with different synchrotron amplitudes rotate at the same rate in phase spisce, mixing of wave phases does not occur. Thus in order to include Jandau damping, nonlinear binding forces must be assumed.

It is clear that in order to include Landau damping all we have to do is to replace $A\left(\theta_{0}, w_{0}, t\right)$ in Eq. (7.2) with $\bar{A}\left(\theta_{0}, w_{0}, t\right)$, the exact $\theta(t)$ for the nonlinear force under consideration. For example, if the potential well is sinusoidal instead of harmonic, $\bar{A}\left(\theta_{0}, w_{0}, t\right)$ is an ellint:c function. ${ }^{15}$ In order to deal with different equilibrium distribution functions, $\psi_{0}\left(\theta_{0}, w_{0}\right)$, one merely makes the replacement

$$
P\left(\theta_{0}\right) \frac{d W\left(w_{0}\right)}{d w} \longrightarrow \frac{1}{M} \frac{\partial w_{0}\left(\theta_{0}, w_{0}\right)}{\partial w}
$$

where $M$ is the normalization constant. Thus in the general case, Eq. (7.4) becomes

$$
I\left(p, r_{1}, s\right)=\frac{1}{(2 \pi)^{2} M} \int_{0}^{\infty} d t e^{-s t} \int d \theta_{0} d w_{0} \frac{\partial \psi_{0}\left(\theta_{0}, w_{0}\right)}{\partial w_{0}} \exp \left\{i r_{1} \theta_{0}-i p \bar{A}\left(\theta_{0}, w_{0}, t\right)\right\}
$$

It is clear that the integrals in Eq. (7.12) are very difficult to do. Therefore, we will present an approximate treatment for the case of cubic nonlinearity. The approximate solution in this case is ${ }^{15}$

$$
\bar{A}\left(\theta_{0}, w_{0}, t\right)=\theta_{0} \cos \Omega_{1} t+\frac{k_{0} w_{0}}{\Omega} \sin \Omega_{1} t
$$


where

$$
\Omega_{1}\left(\theta_{0}, w_{0}\right)=\Omega\left[1-\frac{3 \varepsilon}{8}\left(\theta_{0}^{2}+\frac{k_{0}^{2} w_{0}^{2}}{\Omega^{2}}\right)\right]
$$

and $\varepsilon$ is a nonlinearity parameter. When $\varepsilon \rightarrow 0$, this reduces to the previous solution. Actually, to the same order there are additional terms in liq. (7.13) with frequency $3 \Omega_{1}$. These terms contribute additional resonant terms which are at the same frequencies and are smaller than the resonances already found. We will ignore these additional terms.

Using a Gaussian distribution function, Eq. (7.12) is

$$
I\left(p, r_{1}, s\right)=\frac{1}{(2 \pi)^{2}} \int_{0}^{\infty} d t e^{-s t} Q_{\varepsilon}\left(p, r_{1}, s, t\right)
$$

where

$$
Q_{\varepsilon}\left(p, r_{1}, s, t\right)=\int d \theta_{0} d w_{0} P\left(\theta_{0}\right) \frac{d W\left(w_{0}\right)}{d w} \exp \left\{i r_{1} \theta_{0}-i p\left(\theta_{0} \cos \Omega_{1} t+\frac{k_{0} w_{0}}{\Omega} \sin \Omega_{1} t\right)\right\}
$$

Because $\Omega_{1}=\Omega_{1}\left(\theta_{0}, \mathrm{w}_{0}\right)$, we cannot do this integral. Observe that $Q_{0}\left(p, r_{1}, s, t\right)$ is a periodic function of $\Omega t$. Now, however, the amplitude dependence of $\Omega_{1}$ makes $Q_{\varepsilon}$ aperiodic. $Q_{\varepsilon}$ is a superposition of functions with different frequencies, i.e., a "wave packet" of finite width. As a result, we expect $\mathrm{Q}_{\varepsilon}$ to damp out as a function of time. In fact, a rough asymptotic argument shows that the damping coefficient should be

$$
\delta_{\mathbf{L}} \sim \frac{\mathrm{p}^{\theta_{\mathrm{RMS}}}{ }^{\Delta \Omega_{\mathrm{RMS}}}}{2 \pi}
$$

where $\theta_{\mathrm{PMS}} i$ s the root mean square angular spread in the beam, and $\Delta \Omega_{\mathrm{PMS}}$ is the root riean square synchrotron-frequency spread in the beam. Of course, $\Delta \Omega_{\mathrm{RM}}$ is a function of $\varepsilon$ and $\theta_{\mathrm{RML}}$, which is given by 


$$
\Delta \Omega_{\mathrm{RMS}}=\frac{3 E \Omega \theta_{\mathrm{RMS}}^{2}}{8}
$$

$\delta_{L}$ is the coefficient in the exponentiai camping term. To understand Eq. (7.17), consider a wave with wave number $p$. The time, $T$, for particles to spread one wavelength is given by

$$
\Delta \Omega_{\mathrm{RMS}} \mathrm{T} \sim \frac{2 \pi}{\mathrm{P}}
$$

Since a coherent mode must be confined to the bunch, a packet of sinusoidal waves must exist. There are about $1 / \theta_{\text {RMS }}$ sinusoidal waves in such a packet. Because the energy of a sinusoidal wave will be triansferred to a wave with mode number $p \pm 1$, the time for the packet to damp is

$$
T_{L} \sim \frac{T}{\theta_{R M S}}
$$

Combining Eqs. (7.19) and (7.20), we see that

$$
T_{L}=\frac{1}{\delta_{L}} \sim \frac{2 \pi}{p \theta_{\mathrm{RMS}} \Delta \Omega_{\mathrm{RMS}}}
$$

Equation (7.21) of course only gives the order of magnitude of $\delta_{L}$.

The preceding argment is very crude, and, as a result, we found it necessary to investigate $Q_{\varepsilon}\left(p, r_{1}, s, t\right)$ numerically. In order io put $Q_{\varepsilon}\left(p, r_{1}, s, t\right)$ into a form convenient ior numerical work, let us define

$$
\begin{aligned}
u^{2} & =\theta_{0}^{2}+\frac{k_{0}^{2} w_{0}^{2}}{\Omega^{2}} \\
\phi & =\sin ^{-1} \frac{\theta_{0}}{u}
\end{aligned}
$$


then

$$
\begin{aligned}
& Q_{E}\left(p, r_{1}, s, t\right)=\frac{f^{2} \Omega^{2}}{D \tau} \int_{0}^{\infty} d u u^{2} e^{-\frac{\Omega^{2} r^{2} u^{2}}{2 D T} r^{k^{2}}} \int_{0}^{2 \pi} d \cdot \cos \phi \\
& x \exp \left\{i u \sin \psi_{\psi}\left(r_{1}-p \cos \Omega_{1} t\right)-i u p \cos \theta \sin \Omega_{1} t\right\}
\end{aligned}
$$

The $\phi$ integration can be done, ${ }^{19}$ and we find

$$
\begin{aligned}
Q_{\varepsilon}\left(p, r_{1}, s, t\right) & =-\frac{2 \pi p f^{2} \Omega^{2} i}{D \tau} \int_{0}^{\infty} d u u_{0}^{2} e^{-\frac{\Omega^{2} f^{2} u^{2}}{2 D \tau_{1} k_{0}^{2}}} \\
& \times \frac{\sin \Omega_{1}(u) t}{\sqrt{p^{2}+r_{1}^{2}-2 p r_{1} \cos \Omega_{1}(u) t}} J_{1}\left(u \sqrt{p^{2}+r_{1}^{2}-2 p r_{1} \cos \Omega_{1}(u) t}\right)
\end{aligned}
$$

where $J_{1}$ is the Bessel function of the first kind. Recall that

$$
\Omega_{1}(u)=\Omega\left(1-\frac{3 E u^{2}}{8}\right)
$$

The integral in Eq. $(7.25)$ is very complicated, but it is in a form which is convenient for numerical computation. With L. J. Laslett's help we have progranmed Eq. (7.25) and investigated several cases numerically. Our results can be roughly described as follows: for a couple of damping times, the function is similar to $Q_{0}$ but with an exponential damping. After that, the amplitude of the oscillations remains approximately constant, but the phases of the oscillations shift rather strongly. Since both of these behaviors result in damping when inserted in Eq. (7.15), we assume that $Q_{\varepsilon}$ is adequately represented by 


$$
Q_{\varepsilon}\left(p, r_{1}, s, t\right) \approx e^{-\delta_{L} t} Q_{0}\left(p, r_{1}, s, t\right)
$$

The magnitudes of the maxima, for a typical case, as a function of time are shown in Fig. 1 plotted on log paper. As can be seen, they lie approximately on a straight line. The slope of this line we take to be the damping coefficient. Figure 2 shows the damping coefficient as a function of $\theta_{\text {RMS }}$. Figure 3 shows the damping coefficient as a function of $\Delta \Omega_{\text {PMS }}$. Figure 4 shows the damping coefficient as a function of $p$. In each figure, the straight $l$ ine is a fit to the data. All of our results can be sumarized by the formula

$$
\delta_{\mathrm{L}}=\frac{\mathrm{p}{ }^{\theta_{\mathrm{RMS}}}{ }^{\Delta \Omega_{\mathrm{RMS}}}}{2}
$$

Comparing this with Eq. (7.17), we see that the rough physical argument gave a remarkably accurate result. Examining Eqs. (7.27) and (7.15), we see that Landau danping can be approximately included if we make the substitution

$$
s+s+\delta_{L}
$$

Using (7.29) and (7.10), our integral equation, (6.9), becomes

$$
\begin{aligned}
\tilde{\xi}(p, s) & =\tilde{\sigma}(p, s)-\frac{2 \pi e^{2} f^{3} N i \Omega^{2} n^{2}}{D r_{r} k_{0}\left(\left(s+\delta_{L}\right)^{2}+n^{2} \Omega^{2}\right)} \int d r_{1} \frac{1}{r_{1}} \\
& \times \exp \left\{-\frac{D \tau_{r} k_{0}^{2}\left(p^{2}+r_{1}^{2}\right)}{2 f^{2} \Omega^{2}}\right\} I_{n}\left(\frac{D \tau_{r} k_{0}^{2} r_{1} p}{f^{2} \Omega^{2}}\right) Z\left(-r_{1} \omega_{0}\right) \tilde{\xi}\left(r_{1}, s\right)
\end{aligned}
$$

The singularity at $r_{1}=0$ is only apparent, since $z(0)=0$. 
If we were to expand the kernel of $\mathrm{Eq} .(7.30)$ in a power series in $r_{1}$ and keep only $m$ terms, we would obtain, in the usual way, ${ }^{20} \mathrm{~m}$ possible solutions. Therefore, in general, there are an infinite number of solutions of Eq. $(7,30)$. Taking into account the infinite number of hamonics of $\Omega$, we have a doubly infinite set of coherent oscillations. From Eq. (4.1), we sec that

$$
\frac{D \tau_{r} k_{\theta}^{2}}{f^{2} \Omega^{2}}=\theta_{R M S}^{2}
$$

For wavelengths shorter than, or equal to the bunch length we have

$$
\frac{D \tau_{r_{0}}^{2}}{f^{2} \Omega^{2}} \text { pr } 1>(2 \pi)^{2} \gg 1
$$

Since the arguments of functions in Eq. (7.30) are large, a power series solution converges slowly. We can, however, use the large argunent expansion of the Bessel function, 19

$$
I_{n}(z) \cong \frac{e^{z}}{\sqrt{2 \pi z}}
$$

Using Fq. (7.33) in $(7.30)$, we get

$$
\begin{aligned}
\tilde{\xi}(p, s)= & \tilde{\sigma}(p, s)-\frac{\sqrt{2 \pi} e^{2} f^{4} N i \Omega^{3} n^{2}}{\left(D \tau_{r}\right)^{3 / 2} k_{0}\left|k_{0}\right|\left[\left(s+\delta_{L}\right)^{2}+n^{2} \Omega^{2}\right]} \int d r_{1} \frac{1}{r_{1}} \\
& \times \frac{\exp \left\{-\frac{D \tau_{r} k_{0}^{2}\left(p-r_{1}\right)^{2}}{2 f^{2} \Omega^{2}}\right\}}{\sqrt{p r_{1}}} Z\left(-r_{1} \omega_{0}\right) \tilde{\xi}\left(r_{1}, s\right)
\end{aligned}
$$


All of the approximations which have been used in deriving Eq. (7.34) are usually quite good. For special assumptions about $2\left(\sim_{1} \omega_{0}\right)$, Eq. (7.34) should be easy to solve.

We can simplify Eq. (7.34) considerably if we make one additional approximation. In the short wavelength case, i.e., $\mathrm{P}^{\mathrm{P}} \theta_{\mathrm{RMS}} \gg 2 \pi$, a good approximation is

$$
\exp \left\{-\frac{D \tau_{r} k_{0}^{2}\left(p-r_{1}\right)^{2}}{2 f^{2} \Omega^{2}}\right\}=\sqrt{\frac{2 \pi f^{2} \Omega^{2}}{D \tau_{r} k_{0}^{2}}} \delta\left(p-r_{1}\right)
$$

Using this, Eq. (7.34) becomes

$$
\tilde{\xi}(p, s)=\tilde{\sigma}(p, s)-\frac{2 \pi e^{2} f^{5} N i \Omega^{4} n^{2} Z\left(-p \omega_{0}\right)}{\left(D \tau_{r}\right)^{2} k_{0}^{3}\left[\left(s+\delta_{L}\right)^{2}+n^{2} \Omega^{2}\right] p^{2}} \tilde{\xi}(p, s)
$$

Thus, the dispersion relation is

$$
\left(s+\delta_{L}\right)^{2}=-n^{2} \Omega^{2}-\frac{2 \pi e^{2} f^{5} N i \Omega^{4} n^{2} 2\left(-p \omega_{0}\right)}{\left(D \tau_{T}\right)^{2} p^{2} k_{0}^{3}}
$$

Instability occurs when $\operatorname{Re}(s)>0$. Equation (7.37) is a particularly simple result but should be applied cautiously to real proton beams. Both the treatment of Landau damping and the approximation (7.35) may be inadequate in cases of physical interest. For the longer wavelengths, it is necessary to solve Eq. (7.34).

Let us note that only resistance, i.e., $\operatorname{Re}(z) \neq 0$, can give rise to instability. This agrees with earlier work on this problem. ${ }^{17,18}$ In particular, if we take $\delta_{L}=0$, then any resistance at all causes instab- 
ility. Since landau dalming provides the only stabilization for proton beams, an accurate treatment is necessan;, and the approxithat ion we have presented may not be adequate for detailed applications.

VIII. ELIECTRON BEA'S

In this Section, we derive the dispersion relation for electron beams. We then assume that the waves are stable; i.e. we assuate that diffusion has lengthened the bunch so that the bean is stable. We can then find a formula for bunch length. If the beam, as lengthened by equilibrium effects, is stable, then no turbulent bunch lengthening occurs. Thus, a threshold effect is predicted.

In order to derive the dispersion relation for clectron beanis, we need to know the function $I\left(p, r_{1}, s\right)$ in Eq. (6.10). Remarkably enough, the function, 1 , can be calculated exactly. We present the derivation in Appendix $D$. Let us define

$$
\begin{aligned}
& \omega=\sqrt{\Omega^{2}-\frac{1}{4 \tau_{r}^{2}}}=\Omega \sqrt{1-\frac{1}{4 \tau^{2} \Omega^{2}}} \\
& x=\sin ^{-1} \frac{1}{2 \Omega \tau_{r}}
\end{aligned}
$$

then

$$
\begin{aligned}
I\left(p, r_{2}, s\right) & =\left(\frac{D \tau_{r}\left|k_{0}\right|}{f^{2} \Omega \omega}\right)\left(\frac{i p k_{0}}{2}\right) \exp \left\{\frac{-D \tau_{r} k_{0}^{2}\left(p^{2}+r_{1}^{2}\right)}{2 f^{2} \Omega^{2}}\right\} \\
& \times \int_{0}^{\infty} d t e^{-s t-\frac{t}{2 \tau_{r}}} \sin \omega t \exp \left\{\frac{D \tau_{r} r_{l} p k_{0}^{2}}{f^{2} \Omega \omega} e^{-\frac{t}{2 \tau_{r}}} \cos (\omega t-x)\right\}
\end{aligned}
$$


Let us note that in the 1 imit, $D \rightarrow 0, \tau_{r} \rightarrow \infty, D \tau_{r}$ fixed, Eq. (8.3) goes over into kq. (7.5). (The fact that the very long calculation of Appendix D reduces to the correct result in this special case is a confort.)

We now use the identity ${ }^{19}$

$$
\begin{gathered}
\exp \left\{\frac{D r_{r} r_{1} p_{0}^{2}}{f^{2} \Omega \omega} e^{-\frac{t}{2 \tau_{r}}} \cos (\omega t-x)\right\}=I_{0}\left(\frac{D \tau_{r} k_{0}^{2} r_{I} p}{f^{2} \Omega \omega} e^{-\frac{t}{2 t_{r}}}\right) \\
+2 \sum_{\ell=1}^{\infty} I_{\ell}\left(\frac{D \tau_{r} k_{0}^{2} r_{1} p}{f^{2} \Omega \omega} e^{-\frac{t}{2 \tau_{r}}}\right) \cos \ell(\omega t-x)
\end{gathered}
$$

where $I_{\ell}$ are Bessel functions. Since, in all cases that we are interested in, $\tau_{r} \Omega \gg 1$, we will expand to first order in $1 / 2 \tau \Omega$. In this case

$$
\begin{aligned}
& \omega \simeq \Omega \\
& x \simeq \frac{1}{2 \tau r^{\Omega}}
\end{aligned}
$$

We then have

$$
\begin{aligned}
I\left(p, r_{1}, s\right) & =\left(\frac{D t_{r}\left|k_{0}\right|}{f^{2} \Omega^{2}}\right)\left(\frac{i p k_{0}}{2}\right) \exp \left\{\frac{-D r_{r} k_{0}^{2}\left(p^{2}+r_{1}^{2}\right)}{2 f^{2} \Omega^{2}}\right\} \\
& \times \int_{0}^{\infty} d t e^{-s t-\frac{t}{2 \tau_{r}}} \sin \Omega t\left\{I_{0}\left(\frac{D \tau_{r} k_{0}^{2} r_{1} p}{f^{2} \Omega^{2}} e^{-\frac{t}{2 \tau_{r}}}\right)\right. \\
& \left.+2 \sum_{\ell=1}^{\infty} I_{\ell}\left(\frac{D \tau_{r} k_{0}^{2} r_{l} p}{f^{2} \Omega^{2}} e^{-\frac{t}{2 \tau_{r}}}\right)[\cos \ell \Omega t+\ell x \sin \ell \Omega t]\right\}
\end{aligned}
$$


We have expanded the cosinc. Clearly this requires $\ell x \ll 1$. Because $\tau_{r}$ is usually large compared to the synch otron period, we expect a qualitative behavior similar to the proton beam case. Thus, we expect resonances at multiples of the symchrotron frequency. If we assume $s \approx$ in $\Omega$ and keep only the dominant terms, we get

$$
\begin{aligned}
& J\left(p, r_{1}, s\right)=\left(\frac{D \tau_{r}\left|k_{0}\right|}{f^{2} \Omega^{2}}\right)\left(\frac{i p k_{0}}{2}\right) \exp \left\{\frac{-D \tau r_{0}^{2}\left(p^{2}+r_{1}^{2}\right)}{2 f^{2} \Omega^{2}}\right\} \\
& x \int_{0}^{\infty} d t e^{-s t-\frac{t}{2 \tau r}+i n \Omega t}\left\{\frac { 1 } { 2 \dot { i } } \left[I_{n-1}\left(\frac{D r_{r} k_{0}^{2} r_{1} p}{f^{2} \Omega^{2}} e^{-\frac{t}{2 \tau r}}\right)\right.\right. \\
& \left.-I_{n+1}\left(\frac{D \tau_{r} k_{0}^{2} r_{1} p}{f^{2} \Omega^{2}} e^{-\frac{t}{2 \tau} r}\right)\right]+\frac{x}{2} \\
& \left.\times\left[(n+1) I_{n+1}\left(\frac{D \tau_{r} k_{0}^{2} r_{1} p}{f^{2} \Omega^{2}} e^{-\frac{t}{2 \tau r}}\right)-(n-1) I_{n-1}\left(\frac{D \tau_{r} k_{0}^{2} r_{1} p}{f^{2} \Omega^{2}} e^{-\frac{t}{2 \tau_{r}}}\right)\right]\right\}
\end{aligned}
$$

As discussed previously,

$$
\frac{D \tau_{r_{0}}^{2} r_{1} p}{f^{2} \Omega^{2}} \gg 1
$$

As a result, for times $t<6 \tau_{r}$, the large argument expansion can be used for the Bessel functions, i.e. Eq. (7.31). Thus we have

$$
I_{n}\left(\frac{D \tau_{r} k_{0}^{2} r_{1} p}{f^{2} \Omega^{2}} e^{-\frac{t}{2 \tau} r}\right) \simeq \frac{f \Omega e^{\frac{t}{4 \tau_{r}}}}{\sqrt{2 \pi D \tau_{r} k_{0}^{2} r_{1} p}} \exp \left\{\frac{D \tau_{r} k_{0}{ }^{2} r_{1} p}{f^{2} \Omega^{2}} e^{-\frac{t}{2 \tau_{r}}}\right\}
$$

For times $t<6 \tau_{r}$, this is a good approximation. However, as $t \rightarrow \infty$, 
the left hand side of Eq. (B.B) goes to zero while the right hand side goes to infinity. A better approximation results if we expand the exponential inside the exponential and keep the first two terms. Then

$$
I_{n}\left(\frac{D \tau_{r} k_{0}^{2} r_{2} p e^{-\frac{t}{2 \tau r}}}{f^{2} \Omega^{2}}\right) \simeq \frac{f \Omega e^{\frac{t}{4 \tau_{r}}}}{\sqrt{2 \pi D \tau_{r} k_{0}^{2} r_{1} p}} \exp \left\{\frac{D \tau_{r} k_{0}^{2} r_{1} p}{f^{2} \Omega^{2}}\left(1-\frac{t}{2 \tau}\right)\right\}
$$

For times $t<6 \tau$, Eq. (8.9) is an excellent approximation. Also, as $t+\infty$, both sides of Eq. (8.9) go to zero exponentially. Since there are damping terms much larger than $t / \tau_{r}$, we drop $t / \tau_{r}$ terms from now on. Using Eqs. (7.9) and (8.9), Eq. (8.7) becomes

$$
\begin{aligned}
& I\left(p, r_{1}, s\right) \simeq\left(\frac{D \tau_{r}\left|k_{0}\right|}{f^{2} \Omega^{2}}\right)\left(\frac{i p k_{0}}{2}\right) \exp \left\{\frac{-D \tau_{r} k_{0}^{2}\left(p-r_{1}\right)^{2}}{2 f^{2} \Omega^{2}}\right\} \\
& \times \int_{0}^{\infty} d t \exp \left\{-s t+i n \Omega t-\frac{D k_{0}^{2} r_{1} p}{2 \mathrm{f}^{2} \Omega^{2}} t\right\} \\
& \times\left\{\frac{-\inf ^{3} \Omega^{3}}{\sqrt{2 \pi}\left(D \tau{ }^{k}{ }_{0}^{2} r_{2} p\right)^{3 / 2}}+\frac{x f \Omega}{\sqrt{2 \pi D \tau} r^{k_{0}^{2} r_{2} p}}\right\}
\end{aligned}
$$

Let us note that the diffusion damping term is the same as that derived by Dupree ${ }^{21}$ for a magnetized plasma. Thus, our theory does reproduce the first order results of previous strong turbulence theories.

The integral in Eq. $(8.10)$ is easily performed. If the result is substituted into $\mathrm{Eq}$. (6.9), we obtain 


$$
\begin{aligned}
& \tilde{\xi}(p, s)=\tilde{\sigma}(p, s)-\frac{\pi e^{2} N f^{2} i p k_{0}}{\sqrt{2 \pi D \tau} r_{0}^{2}} \int d r_{1} \frac{\exp \left\{\frac{-D \tau r k_{0}^{2}\left(p-r_{1}\right)^{2}}{2 f^{2} \Omega^{2}}\right\}}{\sqrt{p r_{1}}} \\
& \times \frac{2\left(-r_{1} w_{0}\right) \tilde{\xi}\left(r_{1}, s\right)}{s-i m+\frac{n k_{0}^{2} r_{1} p}{2 f^{2} \Omega^{2}}}\left\{\frac{1}{2 \tau_{r} \Omega}-\frac{i n r^{2} \Omega^{2}}{D \tau_{r} k_{0}^{2} r_{1} p}\right\}
\end{aligned}
$$

Now, if we use the short wavelength approximation, i.e. Eq. (. .35$)$, we get

$$
\tilde{\xi}(p, s)=\tilde{\sigma}(p, s)-\frac{\pi e^{2} N f^{3} i \Omega z\left(-p w_{0}\right) \tilde{\xi}(p, s)}{D \tau_{r} k_{0}\left[s-i n \Omega+\frac{D k_{0}^{2} p^{2}}{2 f^{2} \Omega^{2}}\right]}\left[\frac{1}{2 \tau r \Omega}-\frac{i n f^{2} \Omega^{2}}{D \tau_{r} k_{0}^{2} p^{2}}\right]
$$

Thus, the dispersion relation is

$$
s=\operatorname{in} \Omega-\frac{D k_{0}^{2} p^{2}}{2 f^{2} \Omega^{2}}-\frac{\pi e^{2} N f^{3} i \Omega \Omega\left(-p \omega_{0}\right)}{D \tau_{r}{ }_{0}}\left[\frac{1}{2 \tau_{r} \Omega}-\frac{j n f^{2} \Omega^{2}}{D \tau_{r} k_{0}^{2} p^{2}}\right]
$$

The stability criterion is $\operatorname{Re}(s)<0$. Let us note several things about Eq. (8.13). First, both resistance and inductance can give rise to instability. Previously, only the resistive instability has been fornd, because only proton beams have been treated. For electron beams, an additional dissipative process, namely radiation damping, is prescnt. Thus, inductance, i.e, $\operatorname{Im}(2) \neq 0$, can also causc instability. In fact, the inductive term decreases less rapidly with energy than the resistive term because of the increase of radiation damping. Thus, while the resistive term, considered by Lcbedev, ${ }^{6}$ has a strong dependence on energy, the inductive term has a dependence on energy which is much 
weaker. Let us observe that not only the inaginary part of $z$, but also the real part of $Z$ contributes to a shift in frequency. At high energy, the resistive frequency shift dominates. This suggests that calculations of the equilibrium lengthening effect should take into account a contribution from resistance. Previous theories ${ }^{4}$ have only included the inductive bunch lengthening.

It is clear that it would be difficult to include the effects of Landau damping in the calculation. However, we will assume that Landau damping can be included simply by the substitution

$$
s+s+\delta_{L}
$$

with $\delta_{L}$ given by Eq. $(7.28)$. That this must be approximately correct follows from the fact that the rough physical argument that we gave previously remains unchanged. This treatment of Landau damping is subject to the same criticism as in the proton bean case. However, in th... ase there is another damping mechanism, namely diffusion. In fact, as we will see the diffusion damping usually dominates the Landau damping. As a result, only an estimate of the magnitude of landau damping is needed, and, for this, Eq. (7.28) should be adequate.

The stability criterion is now

$$
\delta_{L}+\frac{D k_{0}^{2} p^{2}}{2 f^{2} \Omega^{2}}>\frac{\pi e^{2} N f^{3} \Omega}{D \tau_{r}\left|k k_{0}\right|}\left[\frac{|\operatorname{Im}(2)|}{2 \tau_{r} \Omega}+\frac{|\operatorname{Re}(z)| n f^{2} \Omega^{2}}{D \tau_{r} k_{0}^{2} p^{2}}\right]
$$

Using Eqs. (7.18), (7.28), and (7.31), this becomes

$$
\frac{3 E \Omega \rho \theta_{\mathrm{RMS}}^{5}}{16}+\frac{\mathrm{p}^{2} \theta_{\mathrm{RMS}}^{4}}{2 \tau \mathrm{r}}=\frac{\pi \mathrm{e}^{2} \mathrm{Nf}\left|\mathrm{k}_{0}\right|}{\Omega}\left[\frac{|\operatorname{Im}(2)|}{2 \tau \mathrm{r}^{\Omega}}+\frac{\mathrm{n}|\operatorname{Re}(\mathrm{z})|}{\mathrm{p}^{2} \theta_{\mathrm{RMS}}^{2}}\right]
$$


Equation (8.16), with equality, gives us a formula for the bunch length. Recall that Eq. (8.16) should be applied only when the bunch length which it predicts is longer than the bunch length as given by the equilibrium theory. If the bunch length that Fq. (8.16) predicts is shorter than that given by equilibrium theory, turbulence is absent.

The equilibrium effect is included in Eq. (8.16), since $\Omega$, and not $\Omega_{0}$, is present. Note that the equilibrium effect does not enter in a simple additive fashion. Recall, by the way, that cquilibrium bunch lenthening occurs when $\Omega<\Omega_{0}$, since this corresponds to a weakening of the binding force.

Finally, let us note that nonlinearities enter into Eq. (8.16) through $\varepsilon$. In our simple model, $E$ is constant. However, if nonlinear distortion of the potential well due to self-fields is significant, then $\varepsilon$ may depend on current. 5

\section{COMPARISON WITH EXPERIMENIS}

In this section, we apply Eq. (8.16) to the experimental data. We first examine the data from SPEAR $I$, and then that from SPEAR II. Finally, we make predictions about turbulent bunch lengthening for PEP.

First, let us note that the ratio of turbulent damping to Landau damping is

$$
\frac{8 \mathrm{p}}{3 \varepsilon \Omega \theta_{\text {PMS }}{ }^{\top} r}
$$

For the cases of interest, this ratio is large, so we will drop the Landau damping term. As a result, our approximate treatment of Landau 
damping should be adequate for this application.

Now we need to specify the impedance function, $2\left(-p \omega_{0}\right)$. Ideally, $Z\left(-p w_{0}\right)$ should be known once the detajls of the machine are known. In practice, $2\left(-\mathrm{p} \omega_{0}\right)$ is not well known. We will assume that

$$
\frac{z\left(-p \omega_{0}\right)}{p}=\text { constant }
$$

This is physically reasonable for a high frequency resonant structure or for the radiation dominated regime. ${ }^{16}$

We also need to specify the wave number $p$. We will assume that

$$
\mathrm{p}_{\mathrm{RMS}}=\text { constant }
$$

If we consider a single mode, as the length of the bunch changes the "size" of the mode changes in proportion. Thus, the wavelengths which make up the mode should be proportional to the bunch length. As a result, we get Eq. (9.3)

We also need an equation for $\Omega$. We will use the expression of Pellegrini and Sessler ${ }^{4}$

$$
\frac{\Omega_{0}^{2}}{\Omega^{2}}=1+\frac{k I}{E^{3} \Delta}
$$

where $I$ is the current in the bunch, $k$ is a constant, $E$ is the beam energy, and $\Delta$ is the bunch length. We will fit the constant, $k$, from the data. As noted earlier, Eq. (9.4) does not include resistanceinduced equilibrium bunch lengthening, however, in the absence of a theory which does, we must use $(9.4)$. For SPEAR II, as we will see, this makes no difturence. Also, for SPEAR I, it might make little difference 
if the $r$ sistance wich contributes to the equilibriun effect is small. Let us also remark that the impedances in. Eq. (9.2), and those contained in the $c$ onstant, $k$, may be quite different, since they correspond to differer: frequencies.

$C\lrcorner r$ formula for the bunch length finally becomes

$$
R^{3}=\left(\frac{\theta_{\text {RNS }}}{\theta_{\text {RMISO }}}\right)^{3}=\frac{2 \pi e^{2} N f\left|k_{0}\right| \tau r}{c \Omega \theta_{\text {RMSO }}^{3}}\left(\frac{A}{2 \tau_{r} \Omega}+B\right)
$$

where $A$ and $B$ are dimensionless constants that we will fit to the data, an $1 \mathrm{c}$ is the speed of light. We have written Eq. (9.5) as a ratio cu ed, where $\theta_{\text {RMSO }}$ is the natural bunch length, i.e.

$$
\theta_{\mathrm{FMSO}}^{2}=\frac{D_{Q} \tau_{r_{0}}^{2}}{\mathrm{f}^{2} \Omega_{0}^{2}}
$$

In Eq. (9.5), there are three constants to fit $-\mathrm{A}, \mathrm{B}$, and the constant $\mathrm{k}$ which onters through Eq. (9.4).

Let us consider the data from SPEAR I. ${ }^{2}$ There are data with three energies, $1.5,2.2$, and $2.5 \mathrm{GeV}$, and with a variety of $\mathrm{rf}$ voltages. It turns out that the bunch lengthening at the higher energies is due entirely to equilibrium effects. Thus, we can determine the constant $k$ from one point at $2.5 \mathrm{GeV}$. The resulting curve is shown in Fig. 5 . The rativ $R$ is plotted as a function of beam current. The solid line is the theoretical curve, the squares are the experimental points. The point with the circle was used to determine $k=.35 \mathrm{GeV}^{3} \mathrm{~ns} . / \mathrm{mA}$. is can he seen, the fit is excellent. We remark that turbulence should begin to contribute at a current of about 285 milliamps (mA). At 2.2 
$\mathrm{CeV}$ there is also no turbulence. The iit is shown in Fig. 6 and is excellent. Turbulence should begin at a current of $220 \mathrm{~mA}$.

At $1.5 \mathrm{GeV}$, turbulence contributes significantly. Two circled points, one in Jig. 7 and one in Fig. 9, were fit to obtain the constants $A$ and $B$, which are given by

$$
\begin{aligned}
& A=2.39 \times 10^{-4} \\
& B=8.6 \times 10^{-8}
\end{aligned}
$$

The fits for various voltages are shown in iigs. 7 through 10 . The dashed lines represent the contribution of the equilibrium effect. As can be seen, the equilibriun contribution is substantial at all voltages. The contribution from turbulence is large at high voltages, but is relatively small at lower voltages. The experimentalists reported $^{2}$ that when half of the ferrite kicker magnets were removed, the bunch lengthening effect was reuuced by about $50^{\circ}$ at lower voltages, but only by about $2 \mathrm{C} 3$ at higher voltages. If we assume that the ferrite kicker magnets contributed to the equilibrium lengthening, but not to the turbulent lengthening, then these observations are perfectly consistent with Figs. 7 through 10 . We remark, by the way, that all of the ferrite magnets were removed in making the transition to SPEAR II.

On SPEAR I, energy widths were calculated by measuring the quantum lifetime of the beam. The results seemed to show only small increases in energy width. Unfortunately, quantum lifetime measurements only reveal the diffusion coefficient at large synchrotron amplitudes, not the energy width of the beam. The energy width is determined by 
the diffusion coefficient near small synchrotron amplitudes, where most of the particles are. It is obvious that the turbulent diffusion coefficient is small at large synchrotron amplitudes, where therc are few particles. Thus the quantum 1 ifetime should be near the natural lifetime even if the cnergy width is increased considerably. Although we have assumed a constant diffusion coefficient in our calculations, it is clear tat it cannot be strictly constant. However, for most of the particles, near the center of the beam, the assimmtion of a constant diffusion coefficient is probably pretty good. The experimental evidence for a nonconstant diffusion coefficient would be a deviation of the particle distribution from a Gaussian at large amplitudes. Since nonlinearity of the potential well also contributes to a non-Gaussian distribution function, it is probably not possible to extract the variation of the diffusion coefficient.

The curve: in Figs. 5 through 10 are not best fits to the data. They were obtained simply by fitting the three circled points. The closeness of the fits is thus an indication that the theory is basically right. Also, we nnte that it is remarkably difficult to obtain good fits with assumptions other than (9.2) and (9.3).

The magnitude of the impedances that (9.7) implies depend on an assumption about wate number, $\mathrm{p}$. With a reasonable assumption, however, we obtain

$$
\frac{\left|\operatorname{Re}\left(Z\left(-\Gamma \omega_{0}\right)\right)\right|}{\Gamma} \sim \frac{\left|\operatorname{Im}\left(z\left(-p \omega_{0}\right)\right)\right|}{p} \sim 1 \text { ohm }
$$

This is a reasonable set of values. ${ }^{22}$

SPEAR II has an $\mathrm{rf}$ system which is considerably different from 
that of SPEAR I. Also, all of the ferrite kicker magnets have been removed. The energy width can now be directly measured by observing the beam width. Thus we are confronted with a different machine and with additional measurements.

The data from SPEAR II are plotted in Figs. il througi $13 .^{3}$ In Figs. 11 and 12, the bunch length is plotted as a function of current. In Fig. 13, the ratio of bunch length to natural bunch iength, dets, and the ratio of energy spread to natural energy spread, crosses, is plotted versus current.

At $3 \mathrm{GeV}$, in Fig. 12, we notice that there seens to be a threshold in current. The equilibrium theories cannot account for such an effect. At $1.5 \mathrm{GeV}$ and $1.7 \mathrm{mV}$, in Fig. 13, we notice that the energy spread ratio and the bunch length ratio seem to be identical. Thus we can conciude that equilibrium effects are negligible for SPEAR II; i.e. we take $k=0$.

Signals from coherent modes can also be measured. With small bunch lengthening, only a few modes are present. As bunch lengthening increases, the number of modes increases. Also, the spectrum looks quite turbulent; i.e, the amplitudes of the modes fluctuate in a seemingly random fashion. Thus, all of the data seems consistent with the interpretation that the bunch lengthening is due to turbulence. The absence of equilibrium bunch lengthening seems natural when it is remembered that all of the ferrite magnets were removed.

Since $k=0$, we have only two constants to fit: $A$ and B. Figures 14 through 18 show the fit to the data. The ratio, $R$, is plotted as a function of the current. The solid lines are the theoretical curves, the squares are the experimental data. The circled points were used to determine $A$ and $B$. The values of these constants are 


$$
\begin{aligned}
& A=4.2 \times 10^{-4} \\
& B=4 . \times 10^{-7}
\end{aligned}
$$

From Figs. 14 and 15, we see that the fits at $1.5 \mathrm{GeV}$ with $1.7 \mathrm{MV}$ and $1 \mathrm{MN}$ are quite good. From Fig. 16, we see that the fit at .6 MV is fairly good, but there seems to be a saturation phenomenon. We will discuss this phenomenon in more detail presently. In Fig. 17, we see that the fit at $3 \mathrm{GeV}$ is pretty good. The points at higher current seem high, but this is probably due to an inadequate control over the rf system, since measurements of the synchrotron frequency varied by about $20 \%$ in this case, but not in the others. In Fig. 18, we see that the threshold for bunch lengthening occurs at about the current at which the measurements stopped. Higher currents have not been achieved yet because of synchtrotron-betatron coupling resonances. In general, the fit to the data is quite good.

The saturation phenomenon at $1.5 \mathrm{GeV}, .6 \mathrm{MV}$, seen in Fig. 16 may only be due to inaccurate measurements. However, if it is an actual phenomenon, there are two possible explanations for it. The first explanation is that it is due to Landau damping. The simple form that we have used, Eq. (8.16), cannot fit the data. This may be due to the fact that our treatment of Landau damping in this model is inadequate. It may also be due to the fact that our model, which does not include nonlinear distortion of the potential well due to self fields, 5 may be inadequate. In the latter case, the saturation is due to the fact that $\varepsilon$, the nonlinearity parameter, is increasing rapidly as a function of current because of the rapidly increasing nonlinearity due to self-fields. In this case, the experimental evidence would be a deviation of the beam 
from a Gaussian shape. However, since a nonconstant diffusion coefficient would also imply a non-Gaussian bean, it is probably difficult to extract the amount of deviation due to nonlinearity.

The second explanation of the saturation phenomenon is that it is due to mode-mode coupling. In other words, the beam stabilizes itself not by increasing its length, but by feeding energy into more and more coherent oscillations. Only a higher order perturbation calculation than ours could predict the contribution of mode-mode coupling. The experimental evidence for mode-mode coupling would consist of the rapid appearance of many higher order coherent oscillations when saturation sets in.

It is possible that both Landau damping and mode-mode coupling contribute to the saturation phenomenon. In any event, it is clear that this is an interesting effect which should be studied in more detail.

Note that the constants given by Eqs. (9.7) and (9.9) are fairly close. Thus while the impedances causing the equilibrium effect were virtually eliminated in SPEAR II, the impedances causing turbulence were increased by a small amount.

In order to apply our theory to PEP, we must estimate the impedances. Let us simple-mindedly just multiply the values of (9.9) by a factor of 10 , since PEP will have 10 times greater circumference than SPEAR. Let us also assume that $k=0$. Using PEP paramete.'s, namely

$$
\begin{aligned}
R & =345 \mathrm{~m} \\
\Omega_{v} & =6.3 \times 10^{4} \mathrm{sec}^{-1} \\
E & =15 \mathrm{GeV} \\
f & =1.38 \times 10^{5} \mathrm{sec}^{-1}
\end{aligned}
$$




$$
\begin{aligned}
\alpha & =.00455 \\
{ }^{\tau} r & =.00823 \mathrm{sec} \\
\theta_{\text {RMSO }} & =8.65 \times 10^{-5}
\end{aligned}
$$

we find

$$
R^{3}=1.1 \times 10^{-2} I^{\prime}
$$

where $I^{\prime}$ is the current in units of milliarms. Thus, $R \geqslant 1$ implies

$$
I>100 \mathrm{~mA}
$$

Since PEP plans to operate at currents-per-bunch less than this, turbulent bunch lengthening should be no problem, if impedances per unit length similar to SPEAR II are attained. 


\section{SUMMARY AND CONCLUSIONS}

We have derived the strong turbulence theory by a Fokker-Planck technique and have investigated the random phase approximation in some detail. Our calculations have shown that we reproduce the first-order results of previous strong turbulence theories.

We have applied our theory to the uniform beam case and have reproduced the earlier result of Sessler. We have also obtained the integral equation for bunched proton beams with general distribution function and potential wells. In the short wavelength case, with a Gaussian distribution function, we were able to obtain a fairly simple dispersion relation for proton beams. The only inadequacy in our treatment of proton beams :vas the approximate analysis of Landau danping.

We were able to obtain the exact integral equation for electrons in a harmonic potential. The dispersion relation in the short wavelength limit was used to obtain a formula for the bunch length. This bunch length formula was applied to the experimental data from SPEAR I and from SPEAR II and good fits to the data resulted from physically reasonable assumptions.

We can conclude that the theory presented in this thesis is adequate to fit almost all of the data. A complete analysis would require solving the integral equation, (8.11), more accurately.

It is clear that more experimental measurements of the saturation phenomenon are needed. More measurements of the energy spread and its correlation with bunch length would allow a more rigorous assessment of the role of turbulence. In particular, energy spread measurements in the saturation regime would help clarify the situation. A detailed study of the coherent modes would also be of use. Observations of the 
bunch shape are needed, although the interpretation of the data would be difficult, because both a nonconstant diffusion coefficient and a nonlinear potential well can affect the bunch shape. Finally, the effect on the bunch length of various parts of the beam environment should be measured, if possible. This would shed light both on the types of impedances which contribute to bunch lengthening and on the possible effects for PEP.

The equilibrium theory should be generalized in two ways. First, the effect of resistive impedances should be included, since it is possible that the resistive effect dominates at high energy. Second, the effect of nonlinearities of the potential well due to self-fields should be studied. Since it seems likely that turbulent bunch lengthening will be negligible at higher energies for PEP, the possible role of equilibrium effects is quite important. Thus, the theory should be developed in more detail.

The contribution of Landau damping should be analyzed much more extensively. This is particularly true for applications to proton beams where Landau damping provides the only stabilization, in linear approximation, of the coherent modes. Also, for electron beams, we have seen that Landau damping may be important in explaining the saturation phenomenon. In particular, the effect on Landau damping of the nonlinear distortion of the potential well due to self-fields should be studied carefully.

The theory of mode-mode coupling should be derived, since it may play an important role in explaining the saturation phenomenon. The theory of mode-mode coupling in uniform plasmas is well known, ${ }^{25}$ but a theory adequate for these applications has not been developed. In fact, 
except for our ignorance of the impedance function, the study of stored particle beams would be an excellent context in which to observe and analyze many nonlinear plasma phenomena. 
APPENDIX A. SOLUTION TO HOMOGENEOUS EQUATION

In this Appendix, we want to derive the solution to Eq. (4.4) that satisfies (4.5). The solution has been obtained previously by Chandrasekhar, ${ }^{12}$ and we reproduce the calculation here merely for the convenience of the reader.

Let us first define

$$
\phi=\frac{\theta}{k_{0}}, \quad B_{1}=\frac{1}{\tau_{r}}, \quad q=\frac{D}{f^{2}}
$$

Using (A1), Eq. (4.4) becomes

$$
\frac{\partial h}{\partial t}+w \frac{\partial h}{\partial \phi}-\left(B_{1} w+\Omega^{2} \phi\right) \frac{\partial h}{\partial w}-B_{1} h=q \frac{\partial^{2} h}{\partial w^{2}}
$$

In order to proceed, we need a lemma. Let $g(t)$ and $f(t)$ be two functions of time. Then the solution of

$$
\frac{\partial F}{\partial t}=f^{2}(t) \frac{\partial^{2} F}{\partial x^{2}}+2 f(t) g(t) \frac{\partial^{2} F}{\partial x \partial y}+g^{2}(t) \frac{\partial^{2} F}{\partial y^{2}}
$$

which has a source at $x=y=0$ when $t=0$, is

$$
F=\frac{1}{2 \pi \Delta^{1 / 2}} \exp \left\{\frac{-\left(a x^{2}+2 l x y+b y^{2}\right)}{2 \Delta}\right\}
$$

where

$$
\begin{aligned}
& a=2 \int_{0}^{t} g^{2}\left(t^{\prime}\right) d t^{\prime} \\
& b=2 \int_{0}^{t} f^{2}\left(t^{\prime}\right) d t^{\prime}
\end{aligned}
$$




$$
\begin{aligned}
& \ell=-2 \int_{0}^{t} g\left(t^{\prime}\right) f\left(t^{\prime}\right) d t^{\prime} \\
& \Delta=a b-l^{2}
\end{aligned}
$$

To prove this lemm, one merely substitutes (A4) into (A3) and uses (A5) through (A8). The calculation is tedious but straightforward. Now let

$$
\begin{aligned}
& \mu_{1}=-\frac{1}{2} \beta_{2}+\sqrt{\frac{1}{4} B_{1}^{2}-\Omega^{2}} \\
& H_{2}=-\frac{1}{2} B_{1}-\sqrt{\frac{1}{4} B_{1}^{2}-\Omega^{2}}
\end{aligned}
$$

If we define new variables

$$
\begin{aligned}
& \xi=\left(\phi \mu_{1}-w\right) \exp \left(-\mu_{2} t\right) \\
& \eta=\left(\phi \mu_{2}-\omega\right) \exp \left(-\mu_{2} t\right)
\end{aligned}
$$

then Eq. (A2) becomes

$$
\frac{\partial h}{\partial t}=\beta_{1} h+q\left\{e^{-2 \mu_{2} t} \frac{\partial^{2} h}{\partial \xi^{2}}+2 e^{-\left(\mu_{1}+\mu_{2}\right) t} \frac{\partial^{2} h}{\partial \xi \partial \eta}+e^{-2 \mu_{1} t} \frac{\partial^{2} h}{\partial \eta^{2}}\right\}
$$

Now, let

$$
G=h e^{\beta_{1} t}
$$

Equation (A13) becomes

$$
\frac{\partial G}{\partial t}=q\left(e^{-2 \mu_{2} t} \frac{\partial^{2} G}{\partial \xi^{2}}+e^{-\left(\mu_{1}+\mu_{2}\right) t} \frac{\partial^{2} G}{\partial \xi \partial \eta}+e^{-2 \mu_{1} t} \frac{\partial^{2} G}{\partial \eta^{2}}\right)
$$


Equation (A15) is of the same form as $\mathrm{Eq}$. (A3). Thus, the solution is

$$
G=\frac{1}{2 \pi \Delta^{\frac{1}{2}}} \exp \left\{-\left[a\left(\xi-\xi_{0}\right)^{2}+2 p\left(\xi_{0}-\xi_{0}\right)\left(n-n_{0}\right)+b\left(\eta-\eta_{0}\right)^{2}\right] / 2 \Delta\right\}
$$

where

$$
\begin{aligned}
& a=\frac{q}{\mu_{1}}\left(1-e^{-2 \mu_{1} t}\right) \\
& b=\frac{q}{\mu_{2}}\left(1-e^{-2 \mu_{2} t}\right) \\
& l=\frac{-2 q}{\mu_{1}+\mu_{2}}\left(1-e^{-\left(\mu_{1}+\mu\right) t}\right)
\end{aligned}
$$

Requiring condition (4.5), we get

$$
\begin{aligned}
h\left(\theta, w, t ; \theta_{0}, w_{0}, 0\right) & =\frac{\left(\sqrt{1 / \tau_{r}^{2}-4 \Omega^{2}}\right) e^{t / \tau_{r}}}{2 \pi\left|k_{0}\right| \sqrt{\Delta(t)}} \exp \left\{-\left[a(t)\left(\xi-\xi_{0}\right)^{2}\right.\right. \\
& \left.\left.+2 \ell(t)\left(\xi-\xi_{0}\right)\left(n-\eta_{0}\right)+b(t)\left(\eta-\eta_{0}\right)^{2}\right] / 2 \Delta(t)\right\}
\end{aligned}
$$


APPENDIX B: UNIFORM BEAM KERNEL

In this Appendix, we calculate the function $\bar{L}(p, s)$ defined by Eq. (5.10). Combining Eqs. (5.10) and (4.11), we find

$$
\bar{L}(p, s)=\frac{1}{2 w} \int_{0}^{\infty} d t e^{-s t} \int d \theta d w d w_{0} \frac{d w\left(w_{0}\right)}{d w} h\left(\theta, w, w_{0}, t\right) e^{-i p \theta}
$$

We now need the function $h$ in the limit $\Omega \rightarrow 0$, Using the notation of Appendix A, we find

$$
\begin{aligned}
\mu_{1} & =0 \\
\mu_{2} & =-\beta_{1} \\
a(t) & =2 q t \\
b(t) & =-\frac{q}{\beta_{1}}\left(1-e^{2 \beta_{1} t}\right) \\
l(t) & =\frac{2 q}{\beta_{1}}\left(1-e^{\beta_{1} t}\right)
\end{aligned}
$$

We thus find that

$$
h=\frac{B_{1} e^{B_{1} t}}{2 \pi \sqrt{\Delta(t)}\left|k_{0}\right|} \exp \left\{-A_{1} \theta^{2}-A_{2} w^{2}-A_{3} w_{0}^{2}-A_{4} \theta w-A_{5} \theta w_{0}-A_{5} w w_{0}\right\} \text { (B3), }
$$

where

$$
\begin{aligned}
& A_{1}(t)=\frac{q \beta_{1}}{2 \Delta k_{0}^{2}}\left(e^{2 \beta_{1} t}-1\right) \\
& A_{2}(t)=\frac{q}{2 \beta_{1} \Delta}\left\{\left(2 \beta_{1} t-3\right) e^{2 \beta_{1} t}+4 e^{\beta_{1}}-1\right\} \\
& A_{3}(t)=\frac{9}{2 \beta_{1} \Delta}\left\{e^{2 \beta_{1} t}-4 e^{\beta_{1} t}+2 \beta_{2} t+3\right\}
\end{aligned}
$$




$$
\begin{aligned}
& A_{4}(t)=\frac{-q}{\Delta k_{0}}\left(e^{\beta_{1} t}-1\right)^{2}=A_{5}(t) \\
& A_{6}(t)=\frac{q}{\beta_{1} \Delta}\left[e^{2 \beta_{1} t}-2 \beta_{1} t e^{\beta_{1} t}-1\right]
\end{aligned}
$$

Recall that

$$
W\left(w_{0}\right)=\exp \left\{-\frac{w_{0}^{2} \beta}{2 q}\right\}
$$

With (B3), we note that the $\theta, w, w_{0}$ integrals in (B1) are Gaussian and are easily done. Doing the $\theta$ and $w$ integrals, we find

$$
\begin{aligned}
\vec{L}(p, s) & =\frac{-\beta_{1}^{2}}{4 \pi q\left|k_{0}\right|} \int_{0}^{\infty} d t \frac{e^{-s t+B_{1} t}}{\sqrt{\Delta(t) A_{2}(t)\left(A_{1}(t)-A_{4}^{2} / 4 A_{2}\right)}} \\
& \times \int d w_{0} w_{0} \exp \left\{-\frac{B_{1} w_{0}^{2}}{2 q}-A_{3} w_{0}^{2}+\frac{A_{6}^{2}}{4 A_{2}} w_{0}^{2}-\frac{p^{2}}{4\left(A_{1}-A_{4}^{2} / 4 A_{2}\right)}\right. \\
& \left.-\frac{2 i p w_{0}\left(-A_{5}+A_{4} A_{6} / 2 A_{2}\right)}{4\left(A_{1}-A_{4}^{2} / 4 A_{2}\right)}+\frac{w_{0}^{2}\left(-A_{5}+A_{4} A_{6} / 2 A_{2}\right)^{2}}{4\left(A_{1}-A_{4}^{2} / 4 A_{2}\right)}\right\} \quad\left(B 10^{\prime} .\right.
\end{aligned}
$$

Using (B4)-(B8), straightforward calculations give

$$
\begin{aligned}
& A_{3}-\frac{A_{6}^{2}}{4 A_{2}}-\frac{\left(-A_{5}+A_{4} A_{6} / 2 A_{2}\right)^{2}}{4\left(A_{1}-A_{4}^{2} / 4 A_{2}\right)}=0 \\
& \frac{-A_{5}+A_{4} A_{6} / 2 A_{2}}{A_{2}-A_{4}^{2} / 4 A_{2}}=\frac{2 k_{0}}{B_{1}}\left(1-e^{-B_{1} t}\right)
\end{aligned}
$$




$$
\begin{aligned}
& \Delta(t) A_{2}(t)\left(A_{1}-\frac{A_{4}^{2}}{4 A_{2}}\right)=\frac{\beta_{1}^{2}}{4 k_{0}^{2}} \cdot e^{2 \beta_{1} t} \\
& \Delta(t)=\frac{q^{2}}{\beta_{1}^{2}}\left(e^{\beta_{1} t}-1\right)\left[e^{\beta_{1} t}\left(2 \beta_{1} t-4\right)+\left(2 \beta_{1} t+4\right)\right] \\
& \frac{1}{4\left(A_{1}-\frac{A_{4}^{2}}{4 A_{2}}\right)}=k_{0}^{2} \frac{q}{2 \beta_{1}^{3}}\left[\left(2 \beta_{1} t-3\right)+4 e^{-B_{1} t}-e^{-2 \beta_{1} t}\right]
\end{aligned}
$$

Thus,

$$
\begin{aligned}
\bar{L}(p, s) & =\frac{-\beta_{3}}{2 \pi q} \int_{0}^{\infty} d t e^{-s t} \exp \left\{\frac{-p^{2} q k_{0}^{2}}{2 \beta_{3}^{3}}\left[\left(2 \beta_{1} t-3\right)+4 e^{-\beta_{1} t}-e^{-2 \beta_{1} t}\right]\right\} \\
& \times \int d w_{0} w_{0} \exp \left\{\frac{-\beta_{1} w_{0}^{2}}{2 q}-\frac{i p w_{0} k_{0}}{\beta_{1}}\left(1-e^{-\beta_{1} t}\right)\right\}
\end{aligned}
$$

Performing the integration over $w_{0}$, we get

$$
\vec{L}(p, s)=i p k_{0} \sqrt{\frac{q}{2 \pi \beta_{1}^{s}}} \int_{0}^{\infty} d x e^{-s x / B_{1}}\left(1-e^{-x}\right) \exp \left\{-\nu\left[x-1+e^{-x}\right]\right\}
$$

where we defined

$$
\begin{aligned}
& \ddot{u}=\beta_{1} t \\
& v=q p^{2} k_{0}^{i} \tau_{r}^{3}
\end{aligned}
$$

Let $y=-x$, and swing the integration contour around in the complex plane from $-\infty$ to $+\infty$. We get 


$$
\bar{L}(p, s)=i p k_{0} \sqrt{\frac{q}{2 \pi \beta_{1}^{5}}} \int_{0}^{\infty} d y e^{s / / \beta_{1}}\left(e^{y}-1\right) \exp \left\{v\left(y+1-e^{y}\right)\right\} \quad \text { (B19). }
$$

Let $x_{1}=e^{y}-1$. Then (B19) becomes

$$
\bar{L}(p, s)=i p k_{0} \sqrt{\frac{q}{2 \pi \beta_{1}^{5}}} \int_{0}^{\infty} d x_{1} x_{1} \exp \left\{-v x_{1}+\left(\frac{s}{\beta_{1}}-1+v\right) \ln \left(x_{1}+1\right)\right\}(B 20) .
$$

Using the definition of $\beta_{1}$ and $q$, this is seen to be identical with Eq. (5.14) . 
APPENDIX C: ALTERNATIVE DERIVATION OF UNIFORM BEAM CASE

In this Appendix, we present another derivation of Eq. (5.16). Sessler ${ }^{7}$ has proviously derived this equation, but, in the course of the calculation, divergent integrals were manipulated. We present a derivation which is similar to Sessler's but which avoids divergent quantities.

For uniform beams $\Omega=0$. Let us assume

$$
\psi(\theta, w, t)=\sum_{n} \psi_{n}(w) e^{i n \theta-i \omega_{n} t}
$$

Then Eq. (2.16) implies

$$
\frac{D}{f^{2}} \frac{d^{2} \psi_{n}}{d w^{2}}+\frac{w}{\tau_{r}} \frac{d \psi_{n}}{d w}+\left(i \omega_{n}-i k_{0} w n+\frac{1}{\tau_{r}}\right) \psi_{n}=-B c e^{2} z_{n} \frac{d \psi_{0}}{d w} \int \psi_{n}(w) d w
$$

where $Z_{n}$ is defined by Eq. (3.30). Let

$$
K_{n}=-\frac{B c e^{2} z_{n} f^{2}}{D} \int \psi_{n}(w) d w
$$

Then Eq. (C2) becomes

$$
\frac{d^{2} \psi_{n}}{d w^{2}}+\left(\frac{f^{2}}{D \tau}\right) w \frac{d \psi_{n}}{d w}+\frac{f^{2}}{D \tau_{r}} \psi_{n}+\frac{i f^{2} \omega_{n}}{D} \psi_{n}-\frac{i n k_{0} f^{2}}{D} w \psi_{n}=K \frac{d \psi_{0}}{d w}
$$

Let

$$
\psi_{n}(w)=\int e^{i w x} \tilde{\psi}_{n}(x) d x
$$

then,

$$
\frac{d \tilde{\psi}_{n}}{d x}+\tilde{\psi}_{n}\left[\frac{-x^{2}+i f^{2} \omega_{n} / D}{\frac{n k_{D} f^{2}}{D}-\frac{x f^{2}}{D \tau_{r}}}\right]=\frac{i \lambda_{0} K_{n}}{2 \pi} \frac{x e^{-D \tau_{r} x^{2} / 2 f^{2}}}{\left(\frac{n k_{0} f^{2}}{D}-\frac{x f^{2}}{D \tau_{r}}\right)}
$$


Equation (C6) is a first order differential equation that we can easily solve. Let

$$
\begin{aligned}
& r(x)=\frac{-x^{2}+\frac{i f^{2} \omega_{n}}{D}}{\frac{n k_{0} f^{2}}{D}-\frac{x f^{2}}{D \tau_{r}}} \\
& g(x)=\frac{i \lambda_{0} K_{n}}{2 \pi} \frac{x e^{-D \tau_{r} x^{2} / 2 f^{2}}}{\left(\frac{n k_{0} f^{2}}{D}-\frac{x f^{2}}{D \tau}\right)}
\end{aligned}
$$

then

$$
\tilde{\psi}_{\mathbf{n}}(x)=\exp \left\{-\int_{\mathbf{a}}^{\mathbf{x}} r\left(x^{\prime}\right) d x^{\prime}\right\} \int_{a}^{x} \exp \left\{\int_{\mathbf{a}}^{\mathbf{x}^{\prime}} r\left(x^{\prime \prime}\right) d x^{\prime \prime}\right\} g\left(x^{\prime}\right) d x^{\prime}+c \exp \left\{-\int_{a}^{x} r\left(x^{\prime}\right) d x^{\prime}\right\}
$$

where a and $\mathrm{C}$ are constants. Recall that

$$
\int d w \psi_{n}(w)=2 \pi \tilde{\psi}_{n}(0)
$$

Thus,

$$
K_{n}=-\frac{\beta c e^{2} z_{n} 2 \pi f^{2}}{D} \tilde{\psi}_{n}(0)
$$

Now,

$$
\begin{aligned}
\int_{a}^{x} r\left(x^{\prime}\right)^{\lambda} x^{\prime} & =\frac{D \tau_{r}}{f^{2}}\left\{-\frac{i f^{2} \omega_{n}}{D} \ln \left(\frac{x-n k_{0}{ } r}{a-n k_{0} \tau_{r}}\right)+\frac{x^{2}}{2}-\frac{a^{2}}{2}\right. \\
& \left.+n k_{0} \tau_{r}(x-a)+n^{2} k_{0}^{2} \tau_{r} \ln \left(\frac{x-n k_{0} \tau r}{a-n k_{0} \tau_{r}}\right)\right\}
\end{aligned}
$$


Thus,

$$
\begin{aligned}
& \tilde{\psi}_{n}(0)=\left(\frac{D \tau_{r}}{f^{2}}\right)\left(\frac{i \lambda_{0} k_{n}}{2 \pi}\right)\left(\frac{1}{n k_{0}{ }^{\tau} r}\right) \int_{a}^{0} d x \times \exp \left\{\frac{\pi k_{0} D \tau_{r}^{2}}{f^{2}} x\right. \\
& \left.+\left(\frac{D n^{2} k_{0}^{2} \tau^{3}}{f^{2}}-i \omega \tau r-1\right) \ln \left(1-\frac{x}{n k_{0} \tau_{r}}\right)\right\} \\
& +C \exp \left\{\frac { - D \tau } { f ^ { 2 } } \left[-\frac{a^{2}}{2}-n k_{0} \tau r^{a}+\left(n^{2} k_{0}^{2} \tau_{r}^{2}-\frac{i f^{2} \omega_{n}}{D}\right)\right.\right. \\
& \left.\left.\times \ln \left(\frac{-n k_{0} \tau}{a-n k_{0} \tau}\right)\right]\right\}
\end{aligned}
$$


In Eq. (C13), we must choose the constants $\mathrm{a}$ and $\mathrm{C}$ so that the answer is finite. Thus, we choose

$$
\begin{array}{llll}
a+\infty, & C=0 & \text { if } & n k_{0}>0 \\
a++\infty, & C=0 & \text { if } & n k_{0}<0 .
\end{array}
$$

With these choices we get for both cases

$$
\begin{aligned}
\tilde{\Psi}_{n}(0) & =\left(\frac{D \tau}{f^{2}}\right)\left(\frac{i \lambda_{0} K n}{2 \pi}\right)\left(\frac{-1}{n k_{0} \tau}\right) \int_{0}^{\infty} \mathrm{dx} x \\
& \times \exp \left\{-\frac{\left|n k_{0}\right| D \tau_{r}^{2} x}{f^{2}}+\left(\frac{D n^{2} k_{0}^{2} \tau^{3} r}{f^{2}}-j \omega \tau \tau^{2}-1\right) \ell n\left(1+\frac{x}{\left|n k_{0}\right| \tau}\right)\right\}
\end{aligned}
$$

Using Eqs. (C11) and (C14) and defining

$$
\begin{aligned}
& y=\frac{x}{\left|n k_{0}\right| \tau_{r}} \\
& v=\frac{n^{2} k_{0}^{2} D \tau_{r}^{3}}{f^{2}} \\
& \mu=i \omega \tau_{r}+1-v
\end{aligned}
$$

the dispersion relation becomes

$$
1=i \lambda_{0} B C e^{2} Z_{n} n k \tau_{0}^{2} \int_{0}^{\infty} d y y \exp \{-v y-\mu \ell n(1+y)\}
$$

Recalling Eq. (5.5), we see that Eqs. (C18) and (5.16) are identical. Sessler derived a dispersion equation which was

$$
1=\frac{i f^{2} \lambda_{0} B C e^{2} Z_{n}}{n k_{0} D \tau_{r}}\left[1-i \omega T_{r} K(\mu, v)\right]
$$


where

$$
K(\mu, \nu)=\int_{0}^{\infty} d y \exp [-v y-\mu \ln (1+y)]
$$

Equations (C18) and (C19) are the same if

$$
\nu \int_{0}^{\infty} d y y e^{-v y-\mu \ln (1+y)}+(\mu+v-1) \int_{0}^{\infty} d y e^{-v y-\mu \ln (1+y)} \stackrel{?}{=} 1
$$

i.e., if

$$
-v \frac{d}{d v}\left(\int_{0}^{\infty} d y e^{-v y-\mu \ln (1+y)}\right)+(\mu+v-1) \int_{0}^{\infty} d y e^{-v y-\mu \ln (1+y)} \stackrel{?}{=} 1
$$

Let $\overline{\mathrm{K}}$ satisfy

$$
-\nu \frac{d \bar{K}(\mu, \nu)}{d \nu}+(\mu+\nu-1) \bar{K}(\mu, \nu)=1
$$

Let us solve Eq. (C23), which is a first order differential equation.

The solution is

$$
\begin{aligned}
\bar{k}(\mu, \nu) & =\int_{a}^{\nu} \exp \left\{-\left(\nu^{\prime}-\nu\right)-(\mu-1) \ln \frac{\nu^{\prime}}{\nu}\right) \frac{-1}{\nu^{\prime}} d \nu^{\prime} \\
& +f(\mu) \exp \left\{\nu-a+(\mu-1) \ln \frac{\nu}{a}\right\}
\end{aligned}
$$

where $a$ is a constant and $f(\mu)$ is a function of $\mu$. Let

$$
r=\frac{\nu^{\prime}}{v}-1
$$

then $(\mathrm{C} 24)$ becomes 


$$
\bar{k}(\mu, \nu)=-\int_{a-\nu}^{0} d r \exp \{-v r-\mu \ln (1+r)\}+f(\mu) \exp \left\{v-a+(\mu-1) \ln \frac{v}{a}\right\}
$$

Choosing $a=\infty, f(\mu)=9$, we see that

$$
\bar{K}(\mu, \nu)=K(\mu, \nu) .
$$

Thus, Eqs. (C18) and (C19) are identical.

Let us finally note that ${ }^{19}$

$$
K(\mu, \nu)=v^{-\mu-1} e^{\nu} \Gamma(\mu+1, \nu)
$$

where $\Gamma(\mu+1, v)$ is an incomplete gama function. 
APPENDIX D: THE FUNCTION I $\left(p, r_{1}, s\right)$

In this Appendix, we derive Eq. (8.3). Using Eqs. (6.12) and (A20), we find, using the notation of Appendix A,

$$
\begin{aligned}
I\left(p, r_{1}, s\right) & =-\frac{f^{2}}{(2 \pi)^{2} D \tau_{r}} \int_{0}^{\infty} d t e^{-s t} \int d \theta_{0} d \theta d w_{0} d w e^{i r_{1} \theta_{0}-i p \theta} w_{0} \\
& \times \exp \left\{-\frac{f^{2} w_{0}^{2}}{2 D \tau_{r}}-\frac{\theta_{0}^{2} f^{2} \Omega^{2}}{2 D \tau_{r} k_{0}^{2}}-B_{1} \theta^{2}-B_{2} \theta_{w}-B_{3} w^{2}\right. \\
& -B_{4} \theta_{0}^{2}-B_{5} \theta_{0} w_{0}-B_{6} w_{0}^{2}-B_{7} \theta \theta_{0}-B_{\theta} \theta_{0}-B_{9} \theta_{0} w \\
& \left.-B_{10} w w_{0}\right\} \times \frac{\sqrt{1 / \tau_{r}^{2}-4 \Omega^{2}}}{2 \pi\left|k_{0}\right| \sqrt{\Delta(t)}} e^{B_{1} t}
\end{aligned}
$$

where

$$
\begin{aligned}
B_{1}(t) & =\frac{1}{2 \Delta k_{0}^{2}}\left[a(t) \mu_{1}^{2} e^{-2 \mu_{2} t}+2 l(t) \mu_{1} \mu_{2} e^{-\left(\mu_{1}+\mu_{2}\right) t}+b(t)\right. \\
B_{2}(t) & =\frac{1}{2 \Delta k_{0}}\left[-2 a(t) \mu_{1} e^{-2 \mu_{2} t}-2 l(t)\left(\mu_{1}+\mu_{2}\right) e^{-\left(\mu_{1}+\mu_{2}\right) t}\right. \\
& \left.-2 b(t) \mu_{2} e^{-2 \mu_{3} t}\right] \\
B_{3}(t) & =\frac{1}{2 \Delta}\left[a(t) e^{-2 \mu_{2} t}+2 l(t) e^{-\left(\mu_{1}+\mu_{2}\right) t}+b(t) e^{-2 \mu_{1} t}\right] \\
B_{4}(t) & =\frac{1}{2 \Delta k_{0}^{2}}\left[a(t) \mu_{1}^{2}+2 l(t) \mu_{1} \mu_{2}+b(t) \mu_{2}^{2}\right]
\end{aligned}
$$




$$
\begin{aligned}
& B_{5}(t)=\frac{1}{2 \Delta k_{0}}\left[-2 \mu_{1} a(t)-2 \ell(t)\left(\mu_{1}+\mu_{2}\right)-2 b(t) \mu_{2}\right] \\
& B_{6}(t)=\frac{1}{2 \Delta}[a(t)+2 l(t)+b(t)] \\
& B_{7}(t)=\frac{1}{2 \Delta k_{0}^{2}}\left[-2 \mu_{1}^{2} a(t) e^{-\mu_{2} t}-2 \ell(t) \mu_{1} \mu_{2}\left(e^{-\mu_{1} t}+e^{-\mu_{2} t}\right)-2 \mu_{2}^{2} b(t) c^{-\mu_{1} t}\right] \\
& B_{\theta}(t)=\frac{1}{2 \Delta k_{0}}\left[2 \mu_{1} a(t) e^{-\mu_{2} t}+2 l(t) \mu_{2} e^{-\mu_{1} t}+2 l(t) \mu_{1} e^{-\mu_{2} t}+2 b(t) \mu_{2} e^{-\mu_{1} t}\right] \\
& B_{g}(t)=\frac{1}{2 \Delta k_{0}}\left[2 a(t) \mu_{2} e^{-\mu_{2} t}+2 l(t) \mu_{1} e^{-\mu_{1} t}+2 \ell(t) \mu_{2} e^{-\mu_{2} t}+2 b(t) \mu_{1} \cdot \mu_{1} t\right] \\
& B_{10}(t)=\frac{1}{2 \Delta}\left[-2 a(t) e^{-\mu_{2} t}-2 l(t)\left(e^{-\mu_{1} t}+e^{-\mu_{2} t}\right)-2 b(t) e^{-\mu_{1} t}\right] \\
& \Delta(t)=\frac{q^{2}}{\mu_{1} \mu_{2}}\left(1-e^{-2 \mu_{1} t}-e^{-2 \mu_{2} t}+e^{2 \beta_{1} t}\right)-\frac{4 q^{2}}{\beta_{1}^{2}}\left(1-2 e^{\beta_{1} t}+e^{2 \beta_{1} t}\right)
\end{aligned}
$$

Looking at Fq. (D1), we see that the $\theta, \theta_{0}, w$, and $w_{0}$ integrals are Gaussian integrals, and can thus be done. Doing first the $w_{0}$ integral, we find 


$$
\begin{aligned}
I\left(p, r_{1}, s\right) & =-\frac{f^{2} \sqrt{B_{1}^{2}-4 \Omega^{2}}}{(2 \pi)^{3}\left|k_{0}\right| D T}-\int_{0}^{\infty} d t \frac{e^{-s t} e^{B_{1} t}}{\sqrt{\Delta(t)}} \sqrt{\frac{\pi}{B_{3}(t)}} \int d w_{0} d \theta_{0} d w_{0} \\
& \times \exp \left\{-\frac{f^{2} w_{0}^{2}}{2 D \tau}+i r_{1} \theta_{0}-i p \theta-\frac{\theta_{0}^{2} f^{2} \Omega^{2}}{2 D \tau r_{0}^{2}}-B_{1} \theta^{2}-B_{4} \theta_{0}^{2}-B_{5} \theta_{0} w_{0}\right. \\
& -B_{6} w_{0}^{2}-B_{7} \theta \theta_{0}-B_{B} \theta_{0}+\frac{B_{2}^{2} \theta^{2}+B_{9}^{2} \theta_{0}^{2}+B_{10}^{2} w_{0}^{2}}{4 B_{3}} \\
& \left.+\frac{B_{2} B_{9} \theta \theta_{0}+B_{2} B_{10} \theta w_{0}+B_{9} B_{10} \theta_{0} w_{0}}{2 B_{3}}\right\}
\end{aligned}
$$

lloing next the $\theta$ integral, we find

$$
\begin{aligned}
& I\left(p, r_{1}, s\right)=-\frac{f^{2} \sqrt{\beta_{2}^{2}-4 \Omega^{3}}}{(2 \pi)^{3}\left|k_{0}\right| D \tau} \int_{0}^{\infty} d t \frac{e^{-s t+\beta_{1} t}}{\sqrt{\Delta(t)}} \sqrt{\frac{\pi}{B_{3}(t)}} \sqrt{\frac{\pi}{B_{1}-B_{2}^{2} / 4 B_{3}}} \\
& \times \exp \left\{\frac{-p^{2}}{4\left(B_{1}-B_{2}^{2} / 4 B_{3}\right)}\right\} \int d w_{0} d \theta_{0} \exp \left\{-\frac{f^{2} w_{0}^{2}}{2 L \tau r}+i r_{1} \theta_{0}\right. \\
& -\frac{\theta_{0}^{2} f^{2} \Omega^{2}}{2 D \tau_{r_{0} k_{0}^{2}}}-B_{4} \theta_{0}^{2}-B_{5} \theta_{0} w_{0}-B_{6} w_{0}^{2}+\frac{B_{9}^{2} \theta_{0}^{2}}{4 B_{3}}+\frac{B_{10}^{2} w_{0}^{2}}{4 B_{3}}+\frac{B_{9} B_{10} \theta_{0} w_{0}}{2 B_{3}} \\
& \tau\left[\theta_{0}^{2}\left(B_{7}-\frac{B_{2} B_{9}}{2 B_{3}}\right)^{2}+w_{0}^{2}\left(B_{8}-\frac{B_{2} B_{10}}{2 B_{3}}\right)^{2}-2 i p \theta_{0}\left(-B_{7}+\frac{B_{2} B_{9}}{2 B_{3}}\right)-2 i p w_{0}\left(-B_{8}+\frac{B_{2} B_{10}}{2 B_{3}}\right)\right. \\
& \left.\left.+2 \theta_{0} w_{0}\left(-B_{7}+\frac{B_{2} B_{9}}{2 B_{3}}\right)\left(-B_{8}+\frac{B_{2} B_{10}}{2 B_{3}}\right)\right] / 4\left(B_{1}-\frac{B_{2}^{2}}{4 B_{3}}\right)\right\}
\end{aligned}
$$

If we select out the $\theta_{0}$ part of the integral, we find 


$$
\begin{aligned}
& \int d \theta_{0} \exp \left\{\operatorname{ir} \theta_{0}-\frac{\theta_{0}^{2} f^{2} \Omega^{2}}{2 D T_{r} k_{0}^{2}}-B_{4} \theta_{0}^{2}-B_{5} \theta_{0} w_{0}+\frac{B_{9}^{2} \theta_{0}^{2}}{4 B_{3}}+\frac{B_{9} B_{10}}{2 B_{3}} \theta_{0} w_{0}\right. \\
& +\frac{\theta_{0}^{2}\left(B_{7}-B_{2} B_{9} / 2 B_{3}\right)^{2}}{4\left(B_{1}-B_{2}^{2} / 4 B_{3}\right)}-\frac{i p \theta_{0}\left(-B_{7}+B_{2} B_{9} / 2 B_{3}\right)}{2\left(B_{1}-B_{2}^{2} / 4 B_{3}\right)} \\
& \left.+\frac{\theta_{0} W_{6}\left(-B_{7}+B_{2} B_{9} / 2 B_{3}\right)\left(-B_{8}+B_{2} B_{10} / 2 B_{3}\right)}{2\left(B_{1}-B_{2}^{2} / 4 B_{3}\right)}\right\} \\
& =\sqrt{\frac{f^{2} \Omega^{2}}{2 D \tau k_{0}^{2}}+B_{4}-\frac{B_{9}^{2}}{4 B_{3}}=\frac{\left(B_{7}-B_{2} B_{9} / 2 B_{3}\right)^{2}}{4\left(B_{1}-B_{2}^{2} / 4 B_{3}\right)}} \exp \left\{\left[-r_{1}^{2}-\frac{p^{2}\left(-B_{7}+B_{2} B_{9} / 2 B_{3}\right)^{2}}{4\left(B_{1}-E_{2}^{2} / 4 B_{3}\right)^{2}}\right.\right. \\
& +W_{0}^{2}\left(-B_{5}+\frac{B_{9} B_{10}}{2 B_{3}}+\frac{\left(-B_{7}+B_{2} B_{9} / 2 B_{3}\right)\left(-B_{8}+B_{2} B_{10} / 2 B_{3}\right)}{2\left(B_{1}-B_{2}^{2} / 4 B_{3}\right)}\right)^{2} \\
& +2 i r_{1} w_{0}\left(-B_{5}+\frac{B_{9} B_{10}}{2 B_{3}}+\frac{\left(-B_{7}+B_{2} B_{9} / 2 B_{3}\right)\left(-B_{8}+B_{2} B_{10} / 2 B_{3}\right)}{2\left(B_{1}-B_{2}^{2} / 4 B_{3}\right)}\right) \\
& +r, p \frac{\left(-B_{7}+B_{2} B_{9} / 2 B_{3}\right)}{\left(B_{1}-B_{2}^{2} / 4 B_{3}\right)}-i p w_{0} \frac{\left(-B_{7}+B_{2} B_{9} / 2 B_{3}\right)}{\left(B_{1}-B_{2}^{2} / 4 B_{3}\right)}\left(-B_{5}+\frac{B_{9} B_{10}}{2 B_{3}}\right. \\
& \left.\left.+\frac{\left(-B_{7}+B_{2} B_{9} / 2 B_{3}\right)\left(-B_{8}+B_{2} B_{10} / 2 B_{3}\right)}{2\left(B_{1}-B_{2}^{2} / 4 B_{3}\right)}\right)\right] \times 1 / 4\left(\frac{f^{2} \Omega^{2}}{2 D \tau} k_{0}^{2}+B_{4}\right. \\
& \left.\left.-\frac{B_{9}^{2}}{4 B_{3}}-\frac{\left(B_{7}-B_{2} B_{9} / 2 B_{3}\right)^{2}}{4\left(B_{1}-B_{2}^{2} / 4 B_{3}\right)}\right)\right\}
\end{aligned}
$$

Using Eqs. ( $(1-17)$ through (A-19), and Eqs. (D2) through (D12), very long but straight forward calculations, give 


$$
\begin{aligned}
& B_{4}-\frac{B_{9}^{2}}{4 B_{3}}-\frac{\left(B_{7}-B_{2} B_{9} / 2 B_{3}\right)^{2}}{4\left(B_{1}-B_{2}^{2} / 4 B_{3}\right)}=0 \\
& -B_{5}+\frac{B_{9} B_{10}}{2 B_{3}}+\frac{\left(-B_{7}+B_{2} B_{9} / 2 B_{3}\right)\left(-B_{8}+B_{2} B_{10} / 2 B_{3}\right)}{2\left(B_{1} \cdot B_{2}^{2} / 4 B_{3}\right)}=0 \quad \text { (D16), } \\
& -B_{6}+\frac{B_{20}^{2}}{4 B_{3}}+\frac{\left(B_{8}-B_{2} B_{10} / 2 B_{3}\right)^{2}}{4\left(B_{1}-B_{2}^{2} / 4 B_{3}\right)}=0
\end{aligned}
$$

thus,

$$
\begin{aligned}
& I\left(p, r_{1}, s\right)=\frac{-f \sqrt{B_{1}^{2}-4 \Omega^{2}}}{(2 \pi)^{2} \Omega \sqrt{2 \pi D \tau_{r}}} \int_{0}^{\infty} d t \frac{e^{-s t+B_{1} t}}{\sqrt{\Delta(t)}}: \frac{-\frac{\pi}{B_{3}(t)}}{\sqrt{\frac{\pi}{B_{1}-B_{2}^{2} / 4 B_{3}}}} \\
& \times \exp \left\{\frac{-p^{2}}{4\left(B_{1}-B_{2}^{2} / 4 B_{3}\right)}-\frac{D \tau r k_{0}^{2} r^{2}}{2 f^{2} S}-\frac{p^{2}\left(-B_{7}+B_{2} B_{9} / 2 B_{3}\right)^{2} D \tau_{r} k_{0}^{2}}{8 f^{2} \Omega^{2}\left(B_{1}-B_{2}^{2} / 4 B_{3}\right)^{2}}\right. \\
& \left.+\frac{D \tau r_{0} k_{0}^{2} r_{1} p\left(-B_{7}+B_{2} B_{9} / 2 B_{3}\right)}{2 f^{2} \Omega^{2}\left(B_{1}-B_{2}^{2} / 4 B_{3}\right)}\right\} \int d w_{0} w_{0} \\
& \times \exp \left\{-\frac{f^{2} w_{0}^{2}}{2 D \tau_{r}}-\frac{\left.i p w_{0} i^{-1}+B_{2} B_{10} / 2 B_{3}\right)}{2\left(B_{1}-B_{2}^{2} / 4 B_{3}\right)}\right\}
\end{aligned}
$$

ibing the integral over $w_{0}$, we find 


$$
\begin{aligned}
& I\left(p, r_{1}, s\right)=\frac{i p D \tau}{r^{\sqrt{\beta_{1}^{2}-4 \Omega^{2}}}} \int_{0}^{\infty} d t \frac{e^{-s t+B_{1} t}}{2(2 \pi)^{2} f^{2} \Omega} \sqrt{\frac{\pi}{\Delta(t)}} \sqrt{\frac{\pi}{B_{3}(t)}} \sqrt{B_{1}-B_{2}^{2} / 4 B_{3}} \\
& \times \frac{\left(-B_{B}+E_{2} B_{10} / 2 B_{3}\right)}{\left(B_{1}-B_{2}^{2} / 4 B_{3}\right)} \exp \left\{\frac{-p^{2}}{4\left(B_{1}-B_{2}^{2} / 4 B_{3}\right)}-\frac{D r r_{0} k_{0}^{2} r_{1}^{2}}{2 f^{2} \Omega^{2}}\right. \\
& -\frac{p^{2}\left(-B_{7}+B_{2} B_{9} / 2 B_{3}\right)^{2} D \tau_{r} k_{0}^{2}}{8 f^{2} \Omega^{2}\left(B_{1}-B_{2}^{2} / 4 B_{3}\right)^{2}}+\frac{D \tau_{r} k_{0}^{2} r_{1} p\left(-B_{7}+B_{2} B_{9} / 2 B_{3}\right)}{2 f^{2} \Omega^{2}\left(B_{1}-B_{2}^{2} / 4 B_{3}\right)} \\
& \left.-\frac{D \tau_{r} p^{2}\left(-B_{8}+B_{2} B_{20} / 2 B_{3}\right)^{2}}{8 f^{2}\left(B_{1}-B_{2}^{2} / 4 B_{3}\right)^{2}}\right\}
\end{aligned}
$$

Using Eqs. (A17) through (A19), and (D2) through (D12), we see that

$$
\begin{gathered}
\frac{\left(-B_{e}+B_{2} B_{10} / 2 B_{3}\right)}{\left(B_{1}-B_{2}^{2} / 4 B_{3}\right)}=\frac{-2}{\mu_{1}-\mu_{2}}\left(e^{\mu_{2} t}-e^{\mu_{1} t}\right) \\
\Delta(t) B_{3}(t)\left(B_{1}-\frac{B_{2}^{2}}{4 B_{3}}\right)=\frac{1}{4}\left(\mu_{1}-\mu_{2}\right)^{2} e^{2 B_{2} t} \\
\frac{\left(-B_{7}+B_{2} B_{9} / 2 B_{3}\right)}{\left(B_{1}-B_{2}^{2} / 4 B_{3}\right)}=\frac{2}{\mu_{1}-\mu_{2}}\left(\mu_{1} e^{\mu_{2} t}-\mu_{2} e^{\mu_{1} t}\right) \\
\frac{1}{4\left(B_{1}-B_{2}^{2} / 4 B_{3}\right)}+\frac{D \tau_{r} k_{0}^{2}\left(-B_{7}+B_{2} B_{9} / 2 B_{3}\right)^{2}}{8 f^{2} \Omega^{2}\left(B_{2}-B_{2}^{2} / 4 B_{3}\right)^{2}}+\frac{D \tau_{r}\left(-B_{8}+B_{2} B_{20} / 2 B_{3}\right)^{2}}{8 f^{2}\left(B_{1}-B_{2}^{2} / 4 B_{3}\right)^{2}}=\frac{D \tau_{r} k_{0}^{2}}{2 f^{2} \Omega^{2}}
\end{gathered}
$$

Using Eqs. (D21) through (D24) in Eq. (D20), we see that 


$$
\begin{aligned}
I\left(p, r_{1}, s\right)= & \frac{D \tau_{r}\left|k_{0}\right|}{f^{2} \Omega \sqrt{\Omega^{2}-\beta_{2}^{2} / 4}} \frac{p k_{0}}{4} \exp \left\{-\frac{D \tau_{r} k_{0}^{2}\left(p^{2}+r_{1}^{2}\right)}{2 f^{2} \Omega^{2}}\right\} \\
& \times \int_{0}^{\infty} d t e^{-s t}\left(e^{\mu_{2} t}-e^{\mu_{1} t}\right) \exp \left\{\frac{D \tau_{r} k_{0}^{2} r_{1} p}{f^{2} \Omega^{2}} \frac{\left(\mu_{1} e^{\mu_{2} t}-\mu_{2} e^{\mu_{1} t}\right)}{\mu_{1}-\mu_{2}}\right\}
\end{aligned}
$$

Using the various definitions, we ser that this is identical with $\mathrm{Eq} .(8\}$.$) .$ 


\section{REFERENCES}

1. F. Amman, Proc. of the 1969 Particle Accelerator Conference, IEEE Trans. Nucl. Sci. NS-16, No. 3, 1073 (1969).

2. M. A. Allen, et al., Proc. of the IX International Conference on High Energy Accelerators, p. 352 (1974).

3. J. M. Paterson, private commication.

4. C. Pellegrini and A. M. Sessler, Nuovo Cimento 3A, p. 116 (1971).

5. E. Keil, PEP Note 126, Stanford Linear Accelerator Center (August 1975), unpub1ished.

6. A. N. Lebedev, Physics with Intersecting Storage Rings, ed. by B. Touschek (Academic Press, New York, 1971).

7. A. M. Sessler, PEP Note 28, Lawrence Berkeley Laboratory (Apri1 1973), unpublished.

8. T. H. Dupree, Phys. of Fluids 9, p. 1773 (1966).

9. J. Weinstock, Phys. of Fluids 11, p. 1977 (1968).

10. L. I. Rudakov and V. N. Tsytovich, P1asma Physics 13, p. 213 (1971).

i1. J. J. Thompson and G. Benford, Phys. Rev. Lett. 28, p. 590 (1972); see also G. Benford and J. J. Thompson, Phys. of Fluids 15, p. 1496 (1972).

12. S. Chandrasekhar, Rev. of Mod. Phys. 15, p. 1 (1943).

13. M. Sands, Physics with Intersecting_Storage Rings, Zoc oit.; or Report No. SLAC-121, Stanford Linear Accelerator Center (1970).

14. B. V. Chirikov, Nucl. Phys. Inst. Report 267 (Novosibirsk, USSR) with English translation by A. T. Sanders, CERN trans. 71-40 (CERN, Geneva, Switzerland; October 1971); G. M. Zaslavskij and B V. Chirikov, Uspekhi Fizicheskikh Nauk $\underline{105}$, and English trans. Sov. l'hys. Usp. 14, p. 549-568 (1972); L. J. Laslett, Proc. of IX 
International Conference on High Energy Accelerators, p. 394 (1974); see also M. A. Lieberman and A. J. Lichtenberg, Phys. Rev. A5, p. 1852 (1972).

15. L. D. Landau and E. M. Lifshitz, Mechanics (Addison-Wesley, Reading, Massachusetts, 1960).

16. A. M. Sessler, Proc. of the 1971 Particle Accelerator Conference, IEEE Trans. Nuc1. Sci. NS-18, No. 3, p. 1039 (June 1971).

17. F. Sacherer, IEEE Trans. Nucl. Sci, NS-20, p. 825 (1973).

18. A. N. Lebedev, VI International Conference on High Energy Accelerators, Cambridge, Massachusetts, p. 284 (1967).

19. I. S. Gradshteyn and I. M. Ryzhik, Tables of Integrals, Series, and Products (Academic Press, New York, New York, 1965).

20. R. Courant and D. Hilbert, Methods of Mathematicai Physics (Wiley Interscience, New York, New York, 1953).

21. T. H. Dupree, Phys. of Fluids 10, p. 1049 (1967).

22. F. Sacherer, PEP Summer Study Note No. 45 (August 1973).

23. J. R. Rees, Proc. of IX International Conference on High Energy Accelerators, p. 564 (1974).

24. R. Z. Sagdeev and A. A. Galeev, Nonlinear Plasma Theory (W. A. Benjamin, New York, New York, 1969). 


\section{FIGURE CAPTIONS}

Fig. 1. Height of maxima of $Q_{\varepsilon}\left(p, r_{1}, s, t\right)$ versus $t$ on a logarithmic scale; $\mathrm{p}=100 ., \Delta \Omega_{\mathrm{RMS}}=.005, \Omega=1 ., \theta_{\mathrm{RMS}} / 2 \pi=.02$.

Fig. 2. $\delta_{\mathrm{L}}$ versus $\theta_{\mathrm{RMS}} / 2 \pi ; \Omega=1, \Delta \Omega_{\mathrm{RMS}}=.005, \mathrm{p}=100$.

Fig. 3. $\delta_{\mathrm{L}}$ versus $\Delta \Omega_{\mathrm{RMS}} ; \quad \Omega=1, \mathrm{p}=100, \theta_{\mathrm{RMS}} / 2 \pi=.04$.

Fig. 4. $\delta_{\mathrm{L}}$ versus $\mathrm{p} ; \quad \Omega=1, \Delta \Omega_{\mathrm{RMS}}=.005, \theta_{\mathrm{RMS}} / 2 \pi=.04$.

Fig. 5. $R$ versus $I$ (current); $E=2.5 \mathrm{GeV}, V_{R F}=475 \mathrm{kV}$; in Figs. 5-10, experimental points are squares, theory is the solid line, circled points were used to fit the theory.

Fig. 6. $R$ versus $I ; E=2.2 \mathrm{GeV}, V_{R F}=300 \mathrm{kV}$

Fig. 7. $R$ versus $I ; E=1.5 \mathrm{GeV}, V_{R F}=525 \mathrm{kV}$; in Figs. $7-10$, the dotted lines show the contribution of the equilibrium theory.

Fig. 8. $R$ versus $I ; E=1.5 \mathrm{GeV}, V_{R F}=275 \mathrm{kV}$.

Fig. 9. $R$ versus $I ; E=1.5 \mathrm{GeV}, V_{R F}=150 \mathrm{kV}$.

Fig. 10. $R$ versus $I ; E=1.5 \mathrm{GeV}, V_{R F}=90 \mathrm{kV}$; the reason for the high values of $R$ at intermediate currents is unknown; it may only be due to lack of experimental control over the RF system.

Fig. 11. Bunch length, $\sigma_{Z}$, versus I; $E=1.5 \mathrm{GeV}, \mathrm{V}_{\mathrm{RF}}=.6,1.0,1.7 \mathrm{mV}$; in Figs. 11-12, circles are experimental points, 1ines are interpolations by hand.

Fig. 12. $\sigma_{2}$ versus $I ; E=3.0 \mathrm{GeV}, V_{R F}=3.0 \mathrm{NN} ; E=3.8 \mathrm{GeV}$, $\mathrm{V}_{\mathrm{RF}}=5.2 \mathrm{MN}$.

Fig. 13. Bunch lengthening, $\sigma_{Z} / \sigma_{Z 0}$, and energy spread increase, $\sigma_{E} / \sigma_{E 0}$, versus I; $E=1.5 \mathrm{GeV}, V_{R F}=1.7 \mathrm{MV}$; the solid line is an interpolation by hand. 
Fig. 14. $R$ versus $I ; E=1.5 \mathrm{GeV}, V_{R F}=1.7 \mathrm{MV}$; in Figs. 14-18, squares are experimental points, solid lines are the theory, circled points were used to fit the constants of the theory.

Fig. 15. $R$ versus $I ; E=1.5 \mathrm{GeV}, V_{R F}=1.0 \mathrm{MV}$.

Fig. 16. $R$ versus $I ; E=1.5 \mathrm{GeV}, V_{R F}=0.6 \mathrm{MN}$; the saturation phenomenon may be due to Landau damping or to mode-mode coupling; it is discussed in the text.

Fig. 17. $R$ versus $I ; E=3.0 \mathrm{GeV}, V_{R F}=3.0 \mathrm{MN}$.

Fig. 18. $R$ versus $I ; E=3.8 \mathrm{GeV}, V_{R F}=5.2 \mathrm{MV}$. 
$-78-$

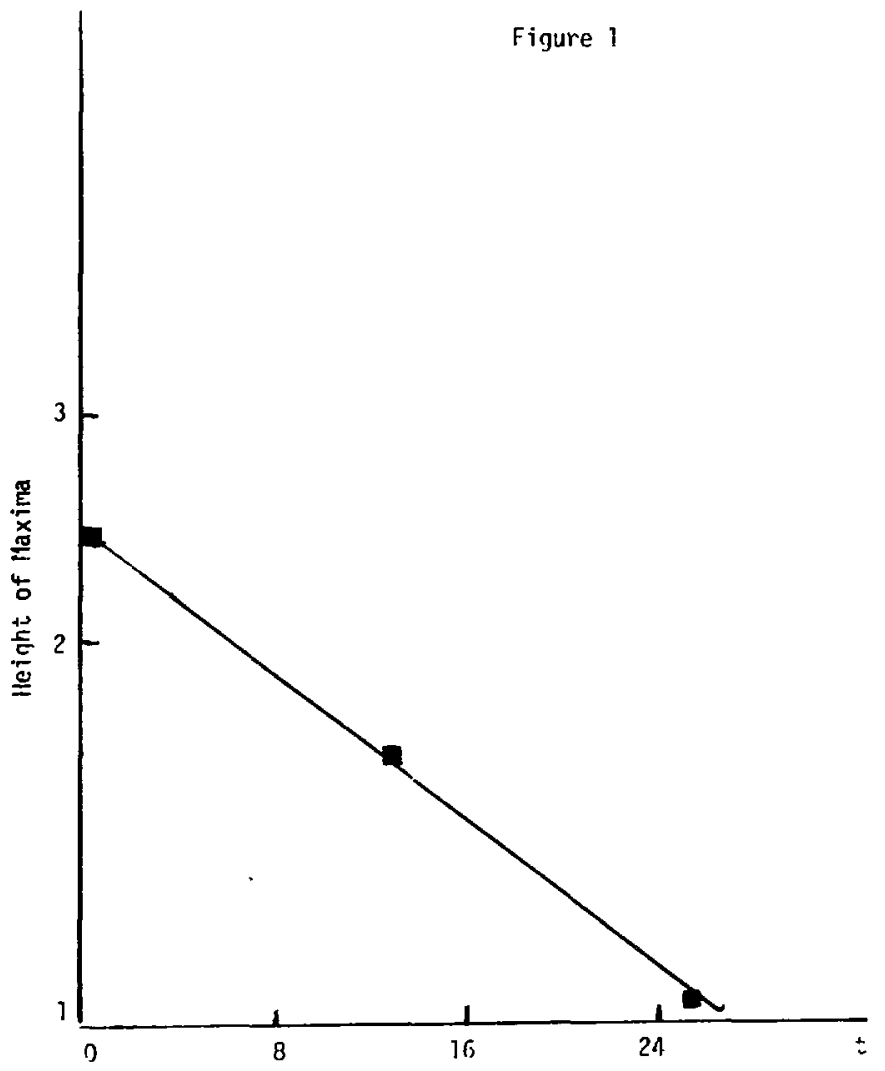


$-79-$

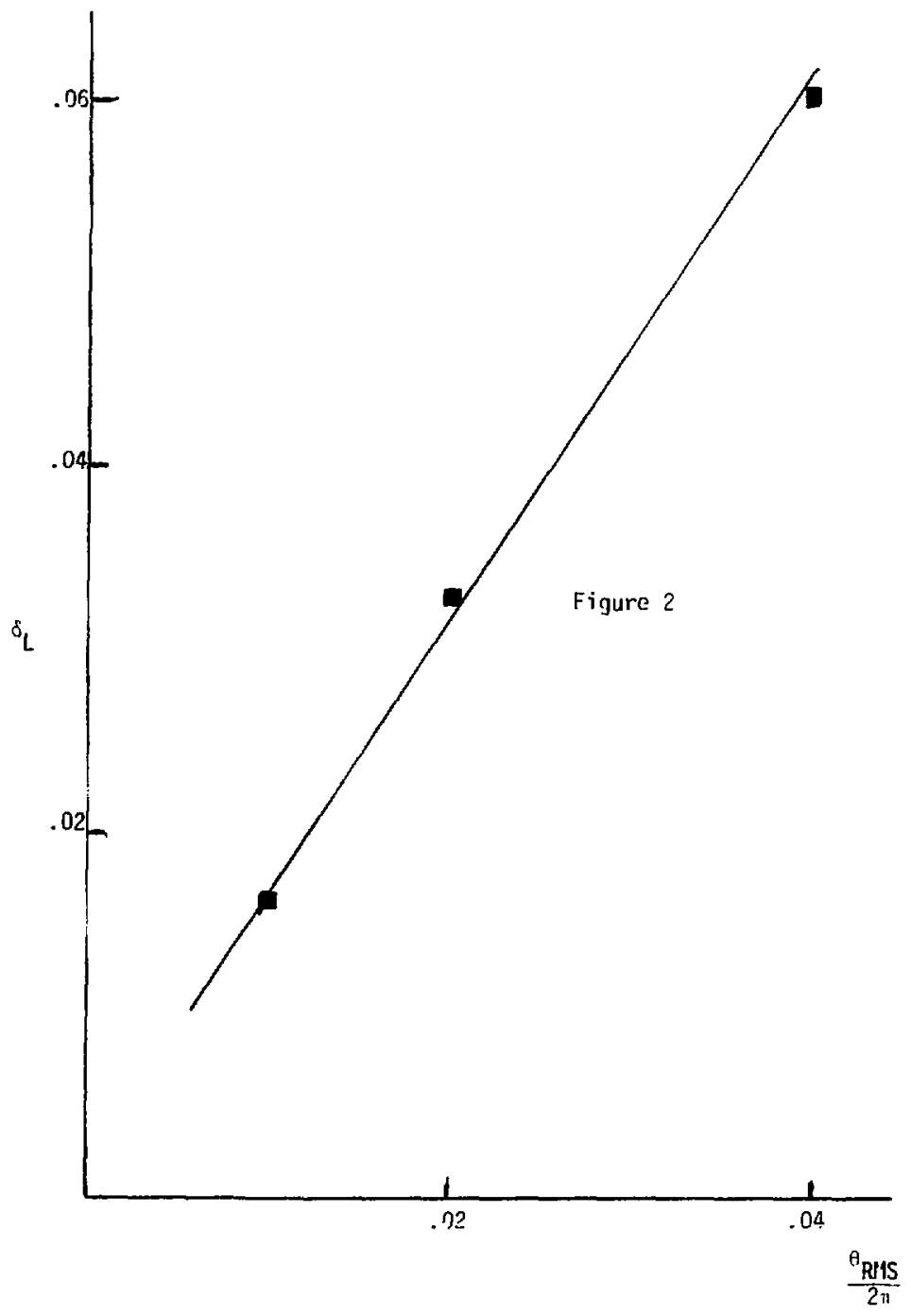


$-80-$

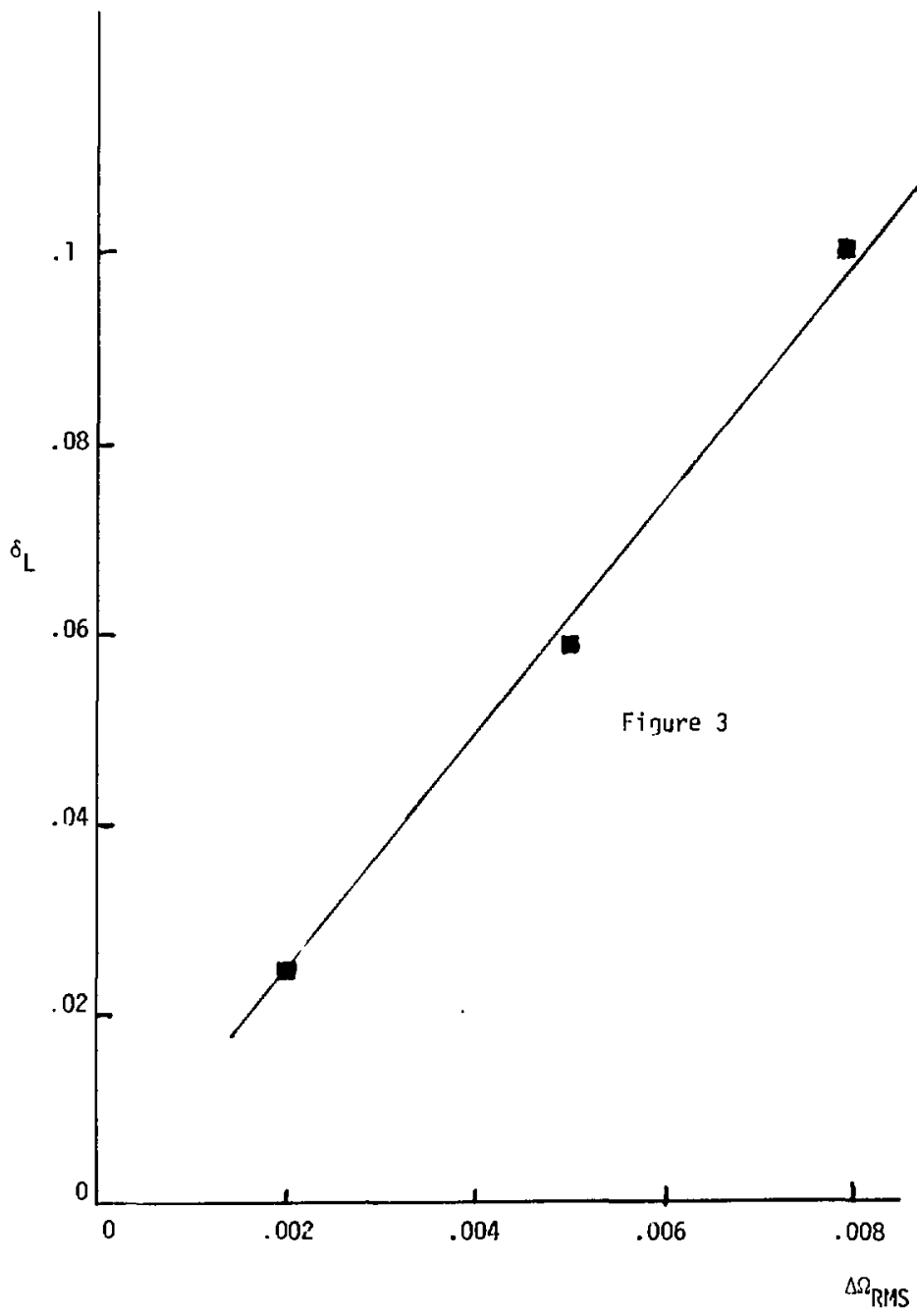


$-81-$

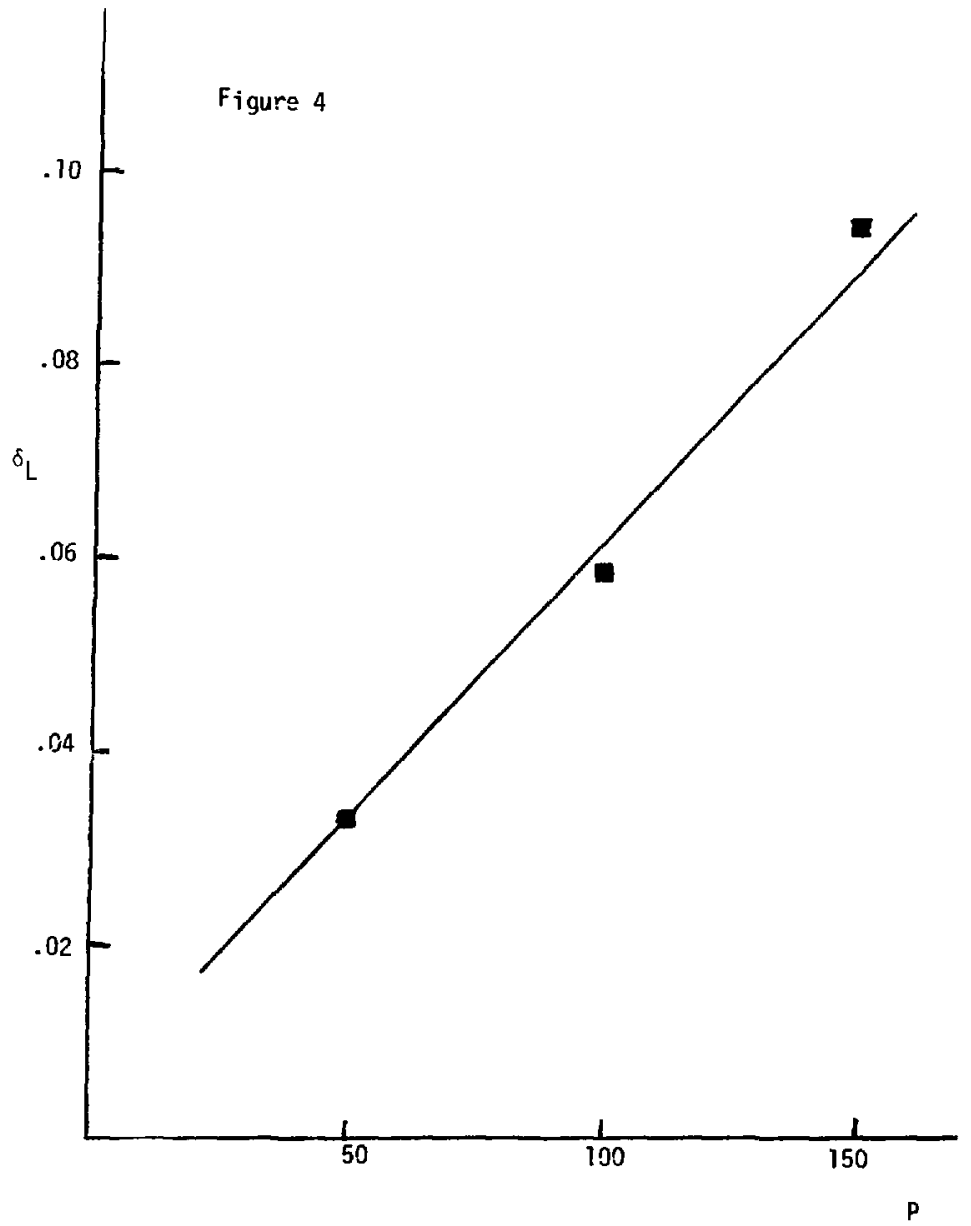


Figure 5

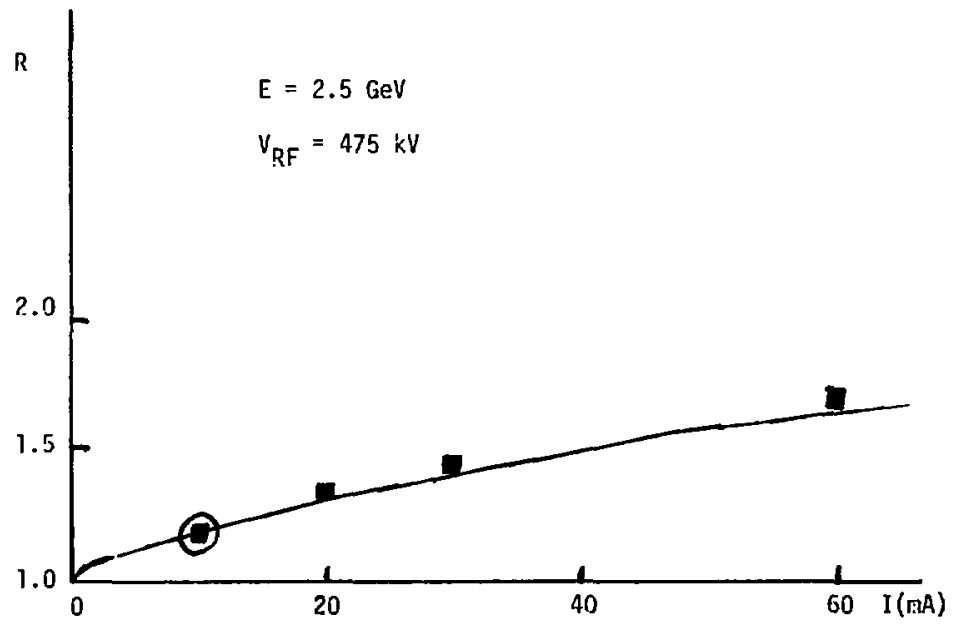




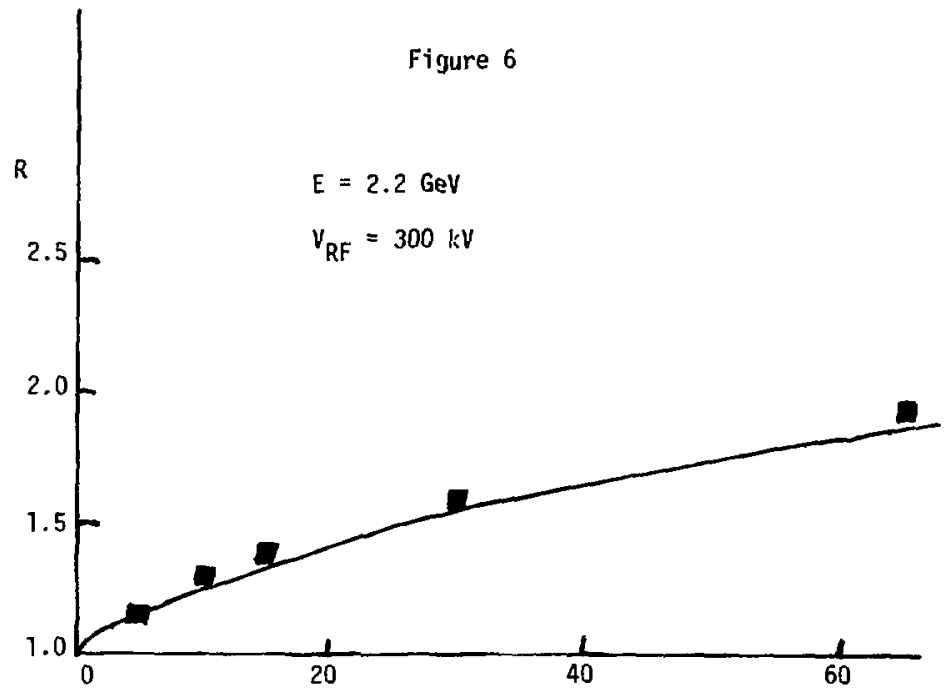


$-84-$

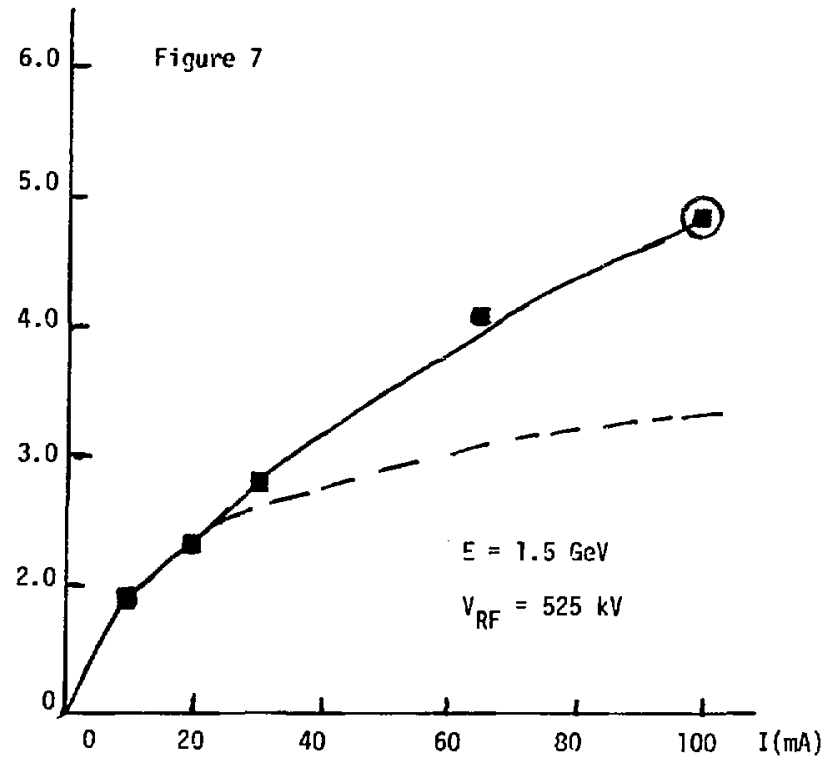




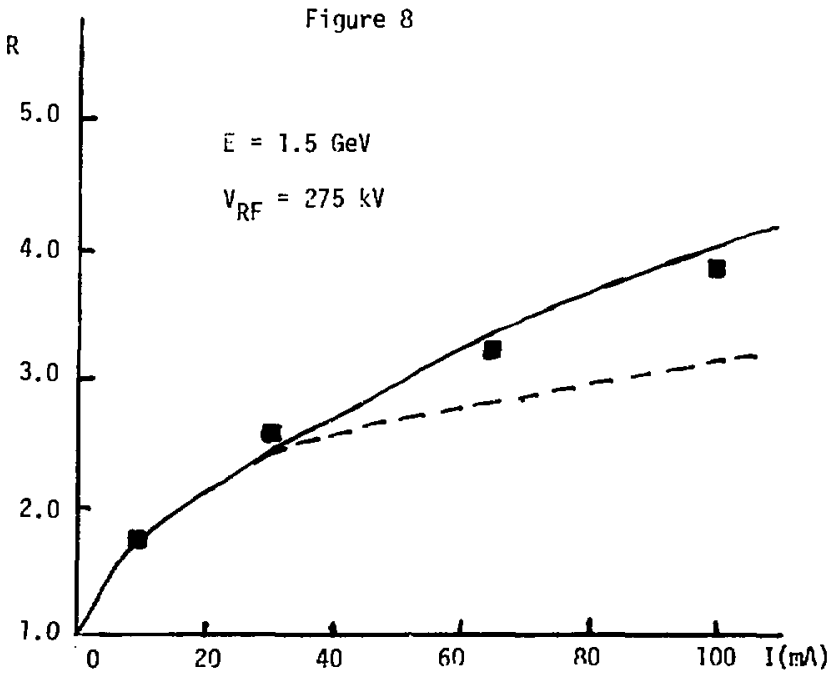




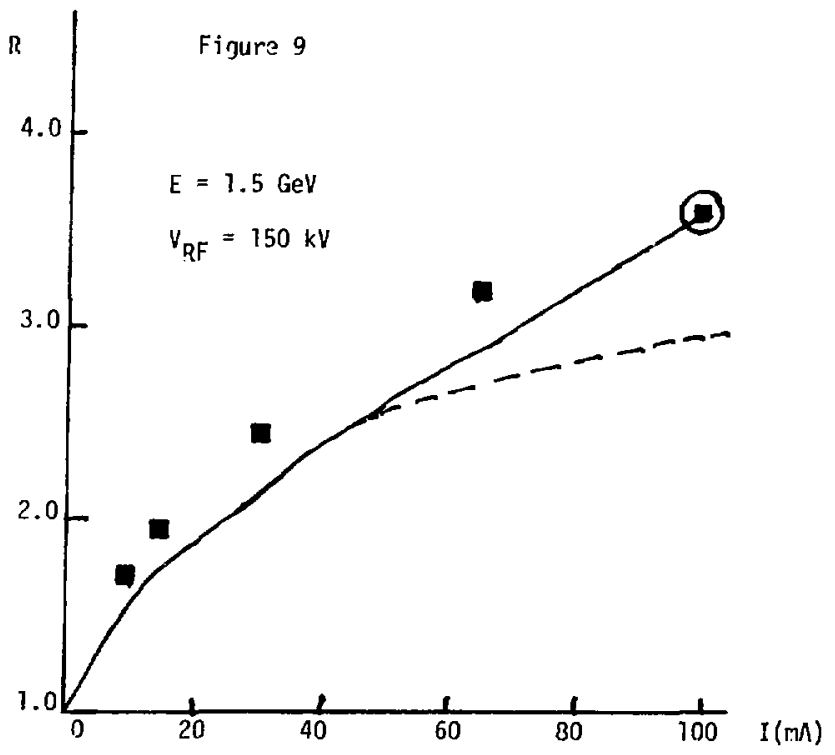


Figure 10

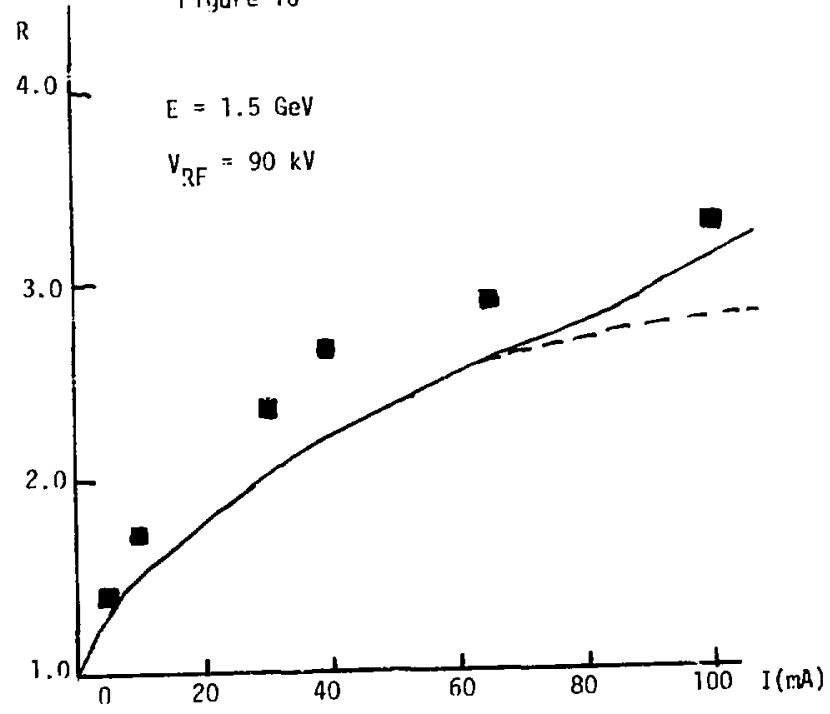




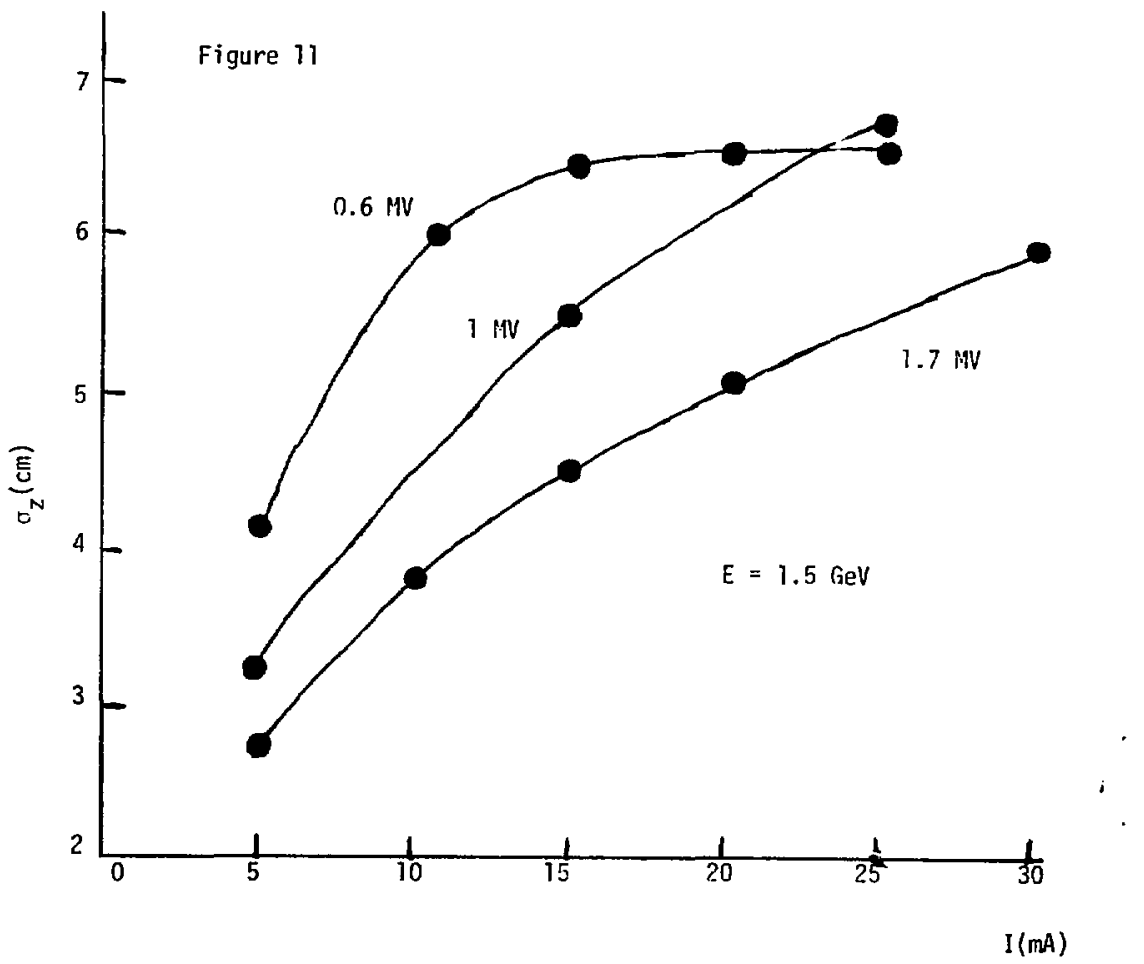




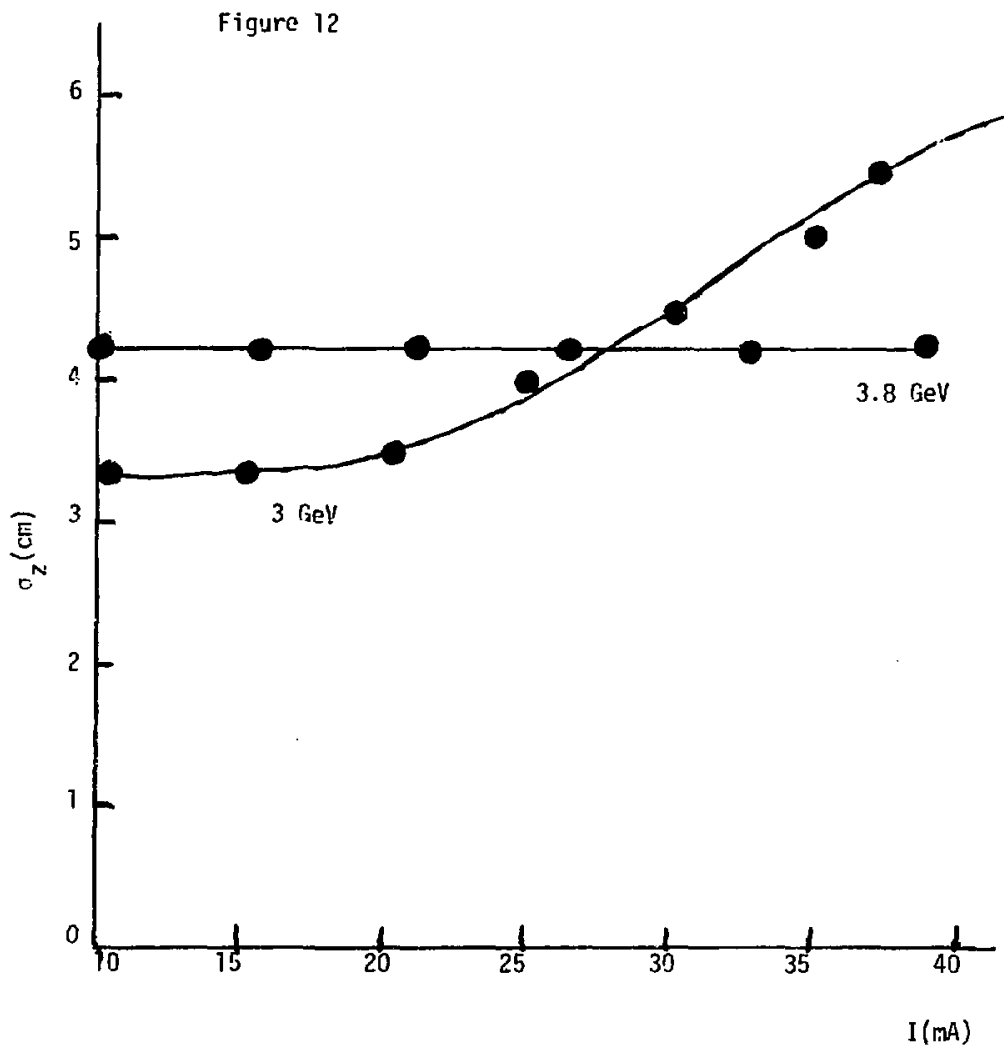




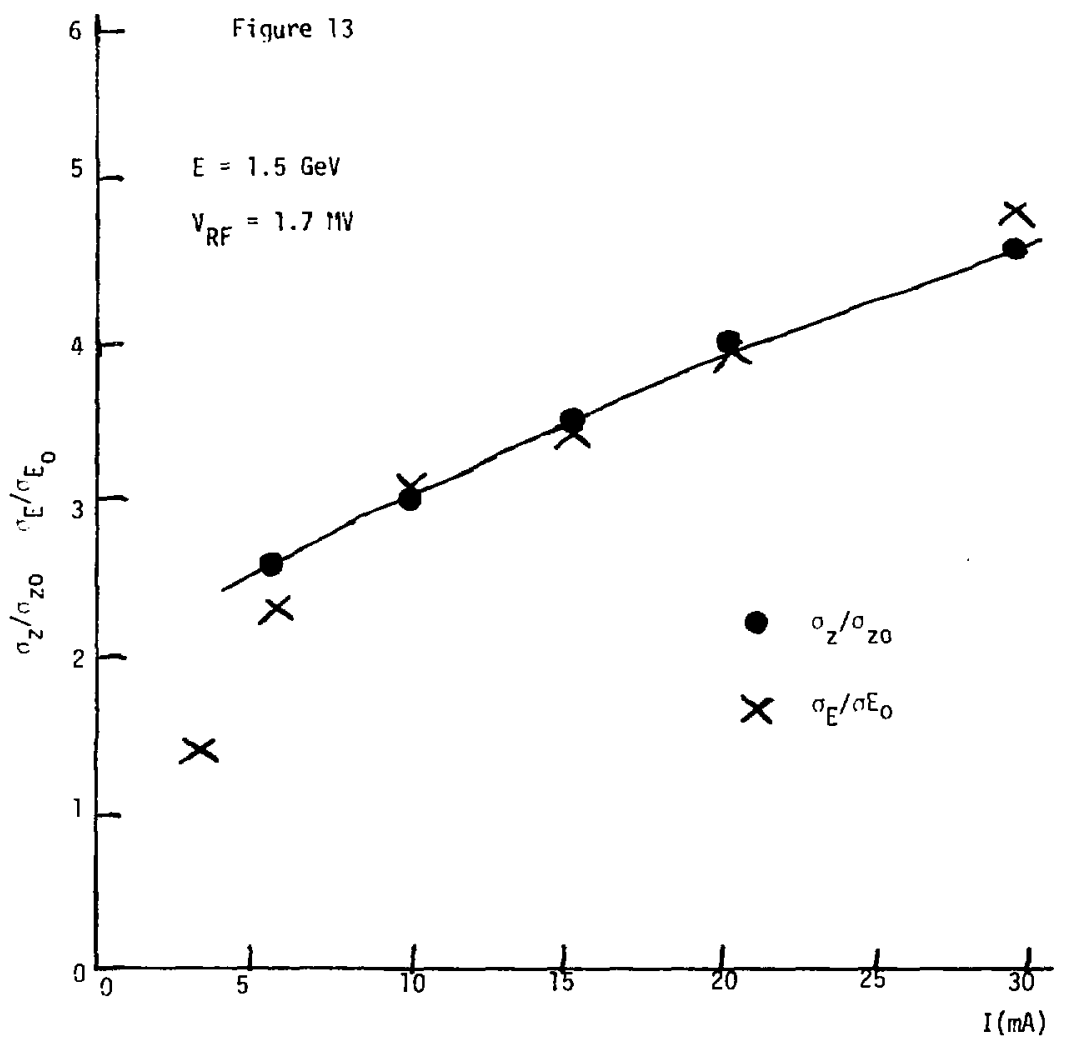




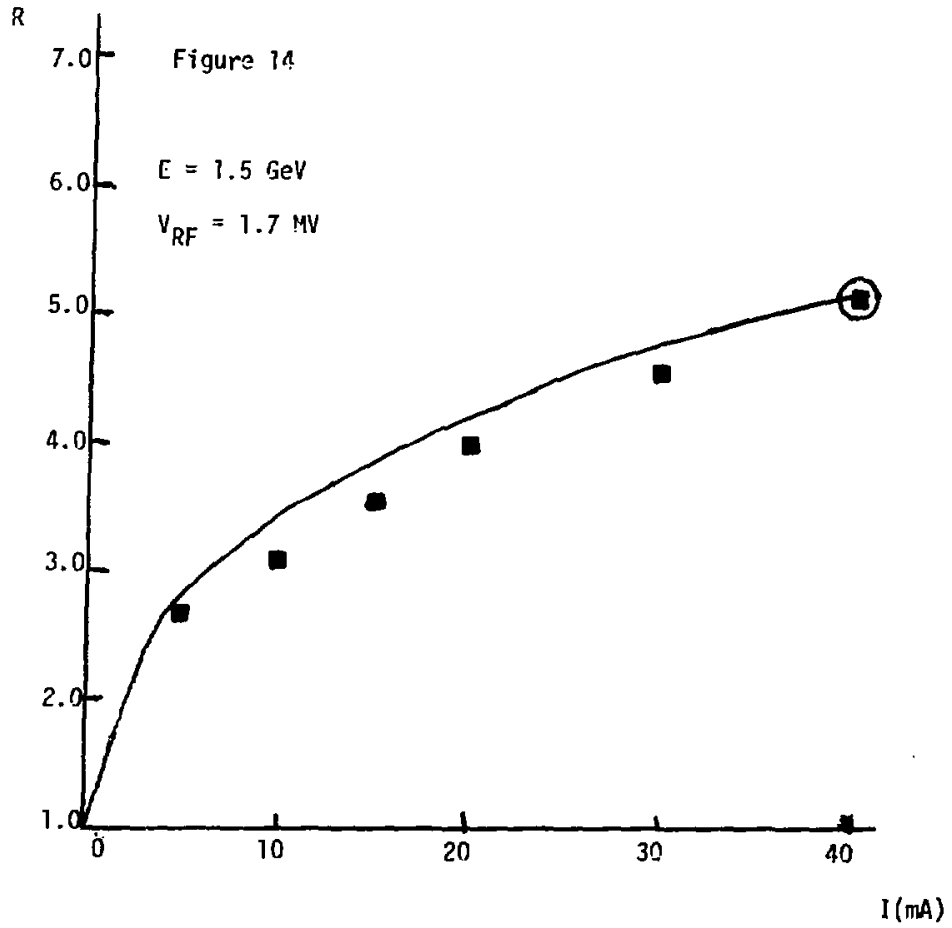




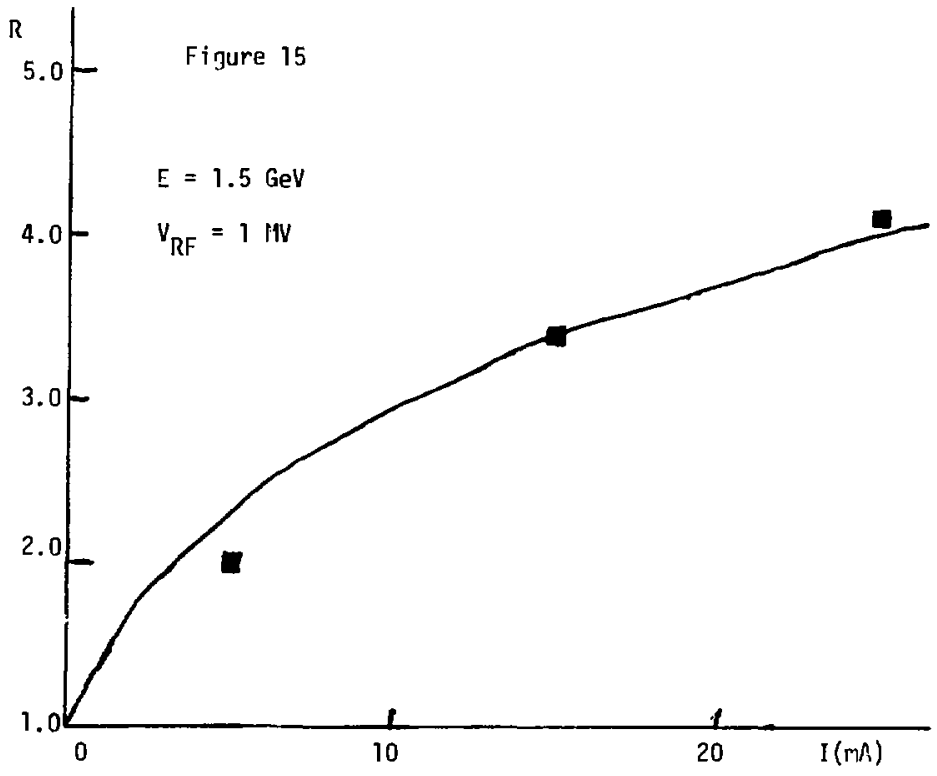


$-93-$

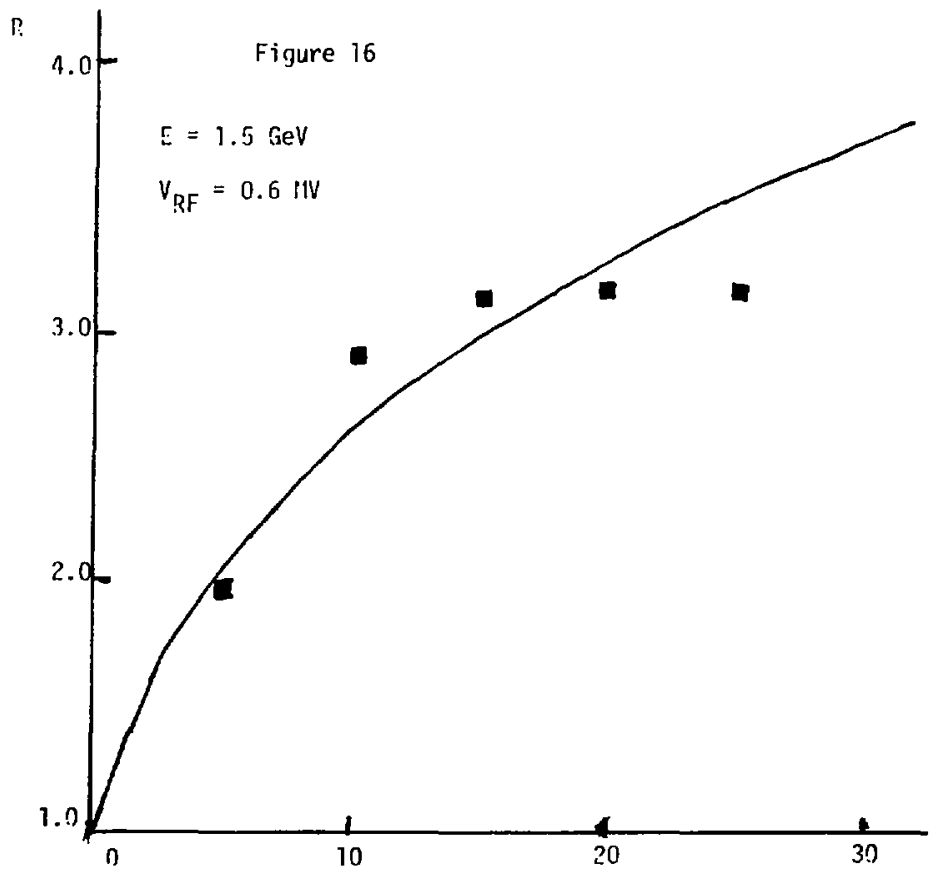




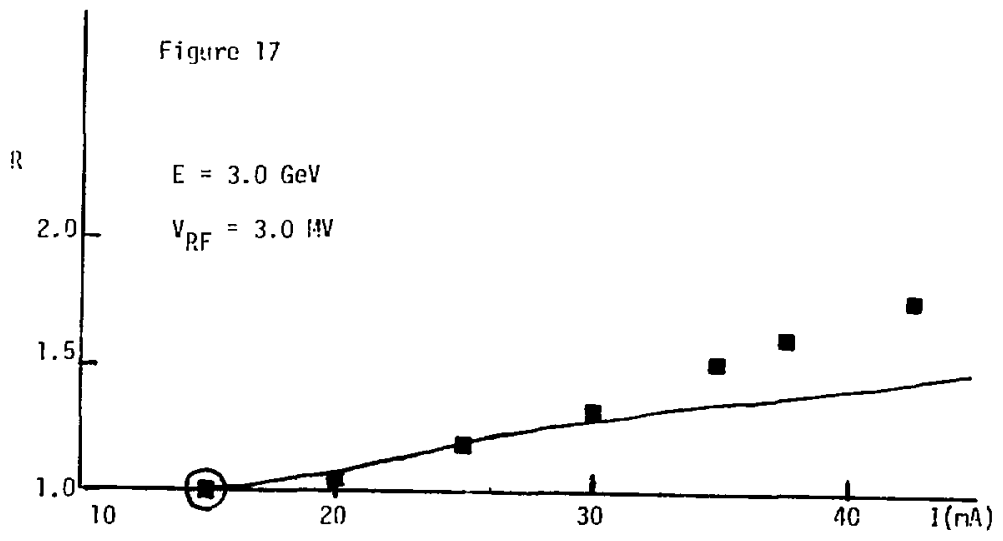




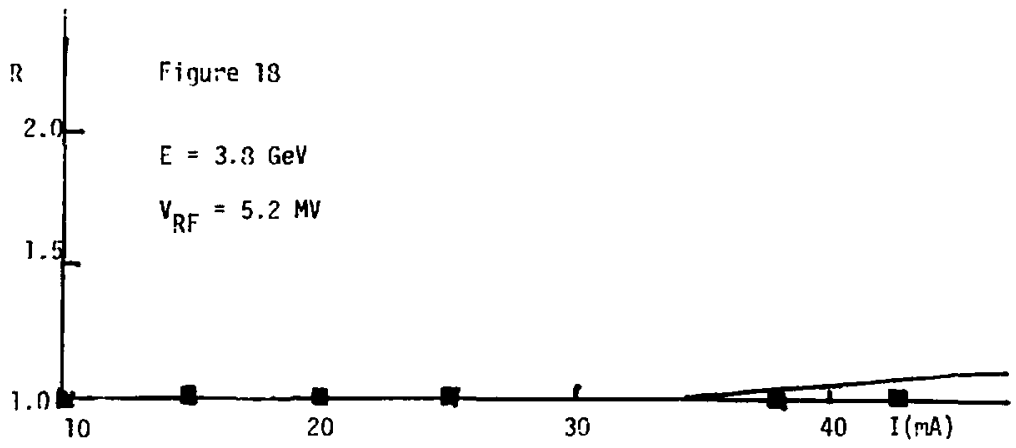

